\title{
Fractional Cointegration In Stochastic Volatility Models
}

\author{
Afonso Gonçalves da Silva*, Peter M. Robinson
}

Department of Economics, London School of Economics and Political Science, Houghton Street, London WC2A 2AE, UK

Discussion paper

No. EM/2007/519

May 2007
The Suntory Centre

Suntory and Toyota International Centres for Economics and Related Disciplines London School of Economics and Political Science Houghton Street London WC2A $2 \mathrm{AE}$ Tel: 02079556679

\footnotetext{
* Address correspondence to Afonso Gonçalves da Silva, Concordia Advisors, Unit 112 Harbour Yard, Chelsea Harbour, London SW10 0XD, UK; e-mail: agsilva@concordiafunds.com.
} 


\begin{abstract}
Asset returns are frequently assumed to be determined by one or more common factors. We consider a bivariate factor model, where the unobservable common factor and idiosyncratic errors are stationary and serially uncorrelated, but have strong dependence in higher moments. Stochastic volatility models for the latent variables are employed, in view of their direct application to asset pricing models. Assuming the underlying persistence is higher in the factor than in the errors, a fractional cointegrating relationship can be recovered by suitable transformation of the data. We propose a narrow band semiparametric estimate of the factor loadings, which is shown to be consistent with a rate of convergence, and its finite sample properties are investigated in a Monte Carlo experiment.
\end{abstract}

Keywords: Fractional cointegration; stochastic volatility; narrow band least squares; semiparametric analysis.

JEL classification: C22 


\section{Introduction}

Financial time series, such as asset returns, are commonly found to be approximately uncorrelated but not independent across time. Much of this dependence can be traced to the fact that volatilities are time dependent, with highly volatile observations grouped in some periods, and relatively low volatilities elsewhere. A great deal of attention has focused on modelling the consequent conditional heteroscedasticity. Influential early contributions were the ARCH model of Engle (1982) (applied there to inflation data), the GARCH extension of Bollerslev (1986), and along a different line, the stochastic volatility (SV) model of Taylor (1986). Empirical evidence has suggested a higher degree of persistence than these models entail, leading to Engle and Bollerslev's (1986) introduction of the IGARCH model. However, the persistence implied by this model (and other unit root based ones, such as IEGARCH) seems too extreme. On the one hand, the absence of mean reversion in the second moments implies permanent shifts to long term volatility forecasts, which is theoretically implausible. On the other, empirical investigation of volatility measures, such as absolute values and squares of observations, suggests they are better explained as stationary processes with long memory, indicating the need for a more flexible model of volatility persistence; see, for example, Whistler (1990), Ding et al. (1993), Ding and Granger (1996), Andersen and Bollerslev (1997).

Several parametric models for this phenomenon have been proposed. Robinson (1991) extended the GARCH framework to an $\operatorname{ARCH}(\infty)$ model that can explain greater persistence. Other models within this framework include Ding and Granger (1996), Baillie et al. (1996), Bollerslev and Mikkelsen (1996). Other authors have extended Taylor's (1986) SV model to explain long memory in squares, e.g. Andersen and Bollerslev (1997), Harvey (1998), Breidt et al. (1998).

In a parallel line of research, asset pricing models assume the existence of one or 
more common factors explaining asset returns. The classical capital asset pricing model (CAPM) of Sharpe (1964) decomposes returns into a single factor, interpreted as the market return, and an idiosyncratic component. The intertemporal CAPM (ICAPM) of Merton (1973) and the arbitrage pricing theory (APT) of Ross (1976) show that, under more realistic assumptions, multiple factors need to be considered as determinants of returns. Estimation of the ICAPM requires correct specification of the factors, there assumed to be observable state variables; the APT uses asymptotics on the cross-sectional dimension (i.e. the number of assets) for its theoretical implications, and also to allow estimation of the unobservable factors and respective loadings. In the present paper, we consider a bivariate model with a single unobservable common factor. It is shown that, under some conditions, persistence in higher moments can allow consistent estimation of the ratio of factor loadings. The bivariate setup is chosen for simplicity; extension of our techniques to more than two observables and one common factor is clearly feasible, but left for future research.

Suppose two observable scalar time series, $y_{t}$ and $x_{t}, t=0, \pm 1, \ldots$, are generated by

$$
\begin{aligned}
& y_{t}=\beta_{1} \zeta_{t}+\varepsilon_{t}, \\
& x_{t}=\beta_{2} \zeta_{t}+\delta_{t},
\end{aligned}
$$

where $\beta_{1}, \beta_{2}$ are unknown, $\beta_{2} \neq 0$, and $\zeta_{t}, \varepsilon_{t}, \delta_{t}$ are unobservable stationary processes, generated by SV models. In asset pricing models, $y_{t}$ and $x_{t}$ would be asset returns, $\zeta_{t}$ could be interpreted as the (unobservable) market return, and $\beta_{1}, \beta_{2}$ would be the market risk exposures of $y_{t}$ and $x_{t}$, respectively. Since the scale of $\zeta_{t}$ cannot be identified, we only aim to estimate $\beta=\beta_{1} / \beta_{2}$; equivalently, $\beta_{2}$ could be normalised to unity by suitably rescaling $\zeta_{t}$. In the suggested interpretation, knowledge of the relative risk exposures of the assets would allow the researcher to compare (and reduce, if necessary) 
the total exposure to market risk of portfolios containing the two assets. In particular, a portfolio could be derived which completely hedges against the common source of risk.

The ordinary least squares (OLS) estimate of $y_{t}$ on $x_{t}$ suffers from errors-in-variables inconsistency for $\beta$, due to the $\delta_{t}$ component in $x_{t}$. Indeed, our assumptions will imply that $\zeta_{t}, \varepsilon_{t}, \delta_{t}$ are white noise sequences (i.e. have zero autocorrelations at all lags), so in no meaningful sense can (1.1), (1.2) be described as a cointegrating relation. However, $\zeta_{t}, \varepsilon_{t}, \delta_{t}$ are not serially independent, but exhibit persistence in higher moments. In particular, for some integer $p>1$, our assumptions imply that $x_{t}^{p}$ and $y_{t}^{p}$ are cointegrated long memory $I(d)$ processes, $0<d<1 / 2$, with cointegrating coefficient $\theta=\beta^{p}$, and cointegrating errors are $I\left(d_{u}\right)$ for $0 \leq d_{u}<d$. Squares of asset returns are typically found to display the underlying persistence, so a cointegrating relationship of the type described could be present, with $p=2$. Still, $x_{t}^{p}$ and $y_{t}^{p}$ are stationary, so the OLS estimate is inconsistent for $\theta$, unlike under the traditional assumption of $I(1)$ observables and $I(0)$ cointegrating errors. The usual instrumental variables estimates employed in time series models (e.g. with $x_{t-1}^{p}$ as instrument) will also be inconsistent here, as the assumed persistence renders all available instruments invalid.

When the spectral density of stationary regressors dominates that of cointegrating errors at low frequencies, Robinson (1994a) showed that a narrow band least squares (NBLS) estimate can be consistent. His observable sequences were linear processes in conditionally homoscedastic martingale difference (md) innovations, which is manifestly not the case in our intrinsically nonlinear framework. Nevertheless, NBLS has been applied to financial data (see e.g. Christensen and Nielsen, 2006; Bandi and Perron, 2006), as have other models for fractional cointegration in volatility (such as the parametric FIGARCH model of Brunetti and Gilbert, 2000), so it would be desirable to establish consistency under more relevant assumptions. The present paper fills this gap in 
the theoretical properties of the NBLS estimate of $\theta$, allowing the latent variables in (1.1), (1.2) to be quite general SV processes. The NBLS estimate converges at a slow (nonparametric) rate, but in long financial series adequate precision may be achievable. Better estimates of $\theta$ are possible (see e.g. Hualde and Robinson, 2006), though they would require at least estimating the memory parameters, and are computationally more intensive and more complicated to handle theoretically. Even for the relatively simple NBLS estimate, our proof of consistency is extremely lengthy.

Note that if we had instead assumed a multiplicative model, where observables are generated by an exponential SV model, and a factor structure is present only in the latent volatility process, a log-squares transformation would yield a linear representation, on which linear process assumptions similar to those of Robinson (1994a) might be plausible (see e.g. Hurvich et al., 2005). In (1.1), (1.2), the presence of additive errors, and the semiparametric SV model which will be introduced, prevent this type of "linearisation." Our specification may be more realistic: common factor structures in the levels often follow from behavioural foundations, as in the CAPM literature, while in volatilities they are typically used just as a convenient assumption for dimensionality-reduction. Furthermore, our approach does not require a specific shape for the volatility function, and indeed allows that shape to vary between the common and idiosyncratic components.

A key component of the proof of consistency is an approximation for expectations of products of nonlinear functions of Gaussian processes (Theorem 1), which may be of independent interest and is presented in the following section, with proof in Appendix A. Section 3 describes the SV setting. Section 4 introduces the NBLS estimate and our consistency result, which is proved in a series of propositions stated and proved in Appendix B, using lemmas in Appendix C, as well as Theorem 1. Sections 5 and 6 consist of a Monte Carlo study of finite sample performance and a discussion of further 
directions for research.

\section{Approximating cross-moments of nonlinear func- tions of Gaussian variables}

With the objective of examining the memory of SV models similar to those introduced in the following section, Robinson (2001) established an asymptotic expansion for the covariance between nonlinear functions of multivariate normal random vectors. Here we need a (non-trivial) extension to cross-moments of more than two real functions.

Let $\phi(\cdot)$ denote the standard normal density and $H_{j}(\cdot)$ the $j$-th Hermite polynomial, for $j \geq 0$, defined by

$$
H_{j}(x) \phi(x)=(-1)^{j} \frac{\partial^{j}}{\partial x^{j}} \phi(x)
$$

For a function $f(\cdot)$ satisfying $\int_{\mathbb{R}} f^{2}(x) \phi(x) \mathrm{d} x<\infty$, define the $j$-th Hermite coefficient $G_{j}=\int_{\mathbb{R}} f(x) H_{j}(x) \phi(x) \mathrm{d} x$ and the Hermite rank $r=\min \left\{j \geq 0: G_{j} \neq 0\right\}$. Define $P_{q}=\{i \in \mathbb{N}: i \leq q\}$, where $\mathbb{N}=\{1,2, \ldots\}, Q_{q}=\left\{(i, j) \in P_{q}^{2}: i<j\right\}$, and $R_{q, k}=\left\{(i, j) \in Q_{q}: i=k\right.$ or $\left.j=k\right\}$ for $k \in P_{q}$.

Theorem 1 For integer $J>1$, let $\mu_{j}, j \in P_{J}$ be jointly normally distributed with zero mean, unit variance, and covariances $\rho_{j k}=\operatorname{Cov}\left(\mu_{j}, \mu_{k}\right), j \neq k$; let $f_{j}=f_{j}\left(\mu_{j}\right)$ be a function such that $E\left(f_{j}^{2}\right)<\infty$, with $k$-th Hermite coefficient $G_{j, k}$ and Hermite rank $r_{j}$. Then

$$
E\left(\prod_{j \in P_{J}} f_{j}\right)=\sum_{q=0}^{\infty} a_{q}
$$

where

$$
a_{q}=\sum_{\substack{v_{\alpha} \geq 0: \\ \Sigma v_{\alpha}=q, \alpha \in Q_{J}}} \prod_{j \in P_{J}} G_{j, w_{j}} \prod_{\alpha \in Q_{J}} \frac{\rho_{\alpha}^{v_{\alpha}}}{v_{\alpha} !}, \quad w_{j}=\sum_{\alpha \in R_{J, j}} v_{\alpha}
$$


If in addition $\tau=2 \sum_{\alpha \in Q_{J}}\left|\rho_{\alpha}\right|<1$, then

$$
\begin{array}{rlrl}
a_{q} & =0 \\
\left|a_{q}\right| & \leq \sigma \prod_{j \in P_{J}}\left(\sum_{\alpha \in R_{J, j}}\left|\rho_{\alpha}\right|\right)^{\frac{r_{j}}{2}} \tau^{q-\frac{r}{2}}, & 2 q<r, \\
\sum_{i=q}^{\infty}\left|a_{i}\right| \leq \sigma \prod_{j \in P_{J}}\left(\sum_{\alpha \in R_{J, j}}\left|\rho_{\alpha}\right|\right)^{\frac{r_{j}}{2}} \frac{\tau^{q-\frac{r}{2}}}{1-\tau}, & 2 q \geq r,
\end{array}
$$

where $r=\sum_{j \in P_{J}} r_{j}$ and $\sigma=\left\{\prod_{j \in P_{J}} E\left(f_{j}^{2}\right)\right\}^{1 / 2}$.

The bounds (2.4), (2.5) reflect the individual, possibly differing, Hermite ranks $r_{j}$ of the $f_{j}$. The weakest version of Theorem 1 arises when $r_{j} \equiv 0$ (i.e. when $E\left(f_{j}\right) \neq 0$ for all $j$ ), and because this would be relevant also when the $r_{j}$ are unknown, we present it in the following Corollary, whose proof follows from the inequality $\sum_{\alpha \in R_{J, j}}\left|\rho_{\alpha}\right| \leq \tau$.

\section{Corollary 1}

$$
\left|a_{q}\right| \leq \sigma \tau^{q}, \quad \sum_{i=q}^{\infty}\left|a_{i}\right| \leq \sigma \frac{\tau^{q}}{1-\tau} .
$$

As in Robinson (2001) in case $J=2$, Theorem 1 provides a valid asymptotic expansion when $\tau \rightarrow 0$. Robinson (1994a) established consistency of the NBLS estimate using $L_{1}$ arguments enabled by linear process (in md innovations) assumptions. Since those are unavailable to us, we use $L_{2}$ arguments. These were also employed by Robinson (1994b) in studying the mean squared error of the averaged periodogram, but in case of Gaussian and linear (in independent and identically distributed, iid, innovations) assumptions. In the SV setting introduced in the following section, matters are considerably more complicated, and we are led to consider various cross-moments of nonlinear functions of Gaussian processes. Theorem 1 is crucial in obtaining sufficiently sharp bounds on these cross-moments to establish consistency. 


\section{Long memory stochastic volatility framework}

To describe the structure of the latent processes $\zeta_{t}, \varepsilon_{t}, \delta_{t}$ in (1.1), (1.2), we first introduce a technical definition of $I(d)$ processes. We say $z_{t}$ is $I(d)$, with memory parameter $d \in[0,1 / 2)$, if it is stationary with finite variance, and has autocovariance function $\rho_{j}=\operatorname{Cov}\left(z_{0}, z_{j}\right)$ satisfying

$$
\sum_{j=0}^{\infty}\left|\rho_{j}\right|<\infty
$$

if $d=0$, and

$$
\begin{aligned}
& \rho_{j} \sim C_{\rho} j^{2 d-1} \text { as } j \rightarrow \infty, \text { for } C_{\rho}>0, \\
& \left|\rho_{j}-\rho_{j+1}\right| \leq K \frac{\left|\rho_{j+1}\right|}{j}, j>0,
\end{aligned}
$$

if $0<d<1 / 2$, where $K$ throughout denotes a generic, arbitrarily large finite constant, and the symbol " " indicates that the ratio of left- and right-hand sides tends to one. We say an $I(0)$ process has short memory, and an $I(d)$ process, for $0<d<1 / 2$, has long memory. We can deduce from (3.1) or (3.2), (3.3) properties of the spectral density $f(\lambda)$ of $z_{t}$, which satisfies $\rho_{j}=\int_{-\pi}^{\pi} f(\lambda) \cos (j \lambda) \mathrm{d} \lambda$. For $d=0, f(\lambda)$ is continuous for all $\lambda$, whereas for $0<d<1 / 2$, Theorem III-12 of Yong (1974) indicates that

$$
f(\lambda) \sim C_{f} \lambda^{-2 d} \text { as } \lambda \rightarrow 0^{+}
$$

where

$$
C_{f}=\pi^{-1} \Gamma(2 d) \sin \left\{(1-2 d) \frac{\pi}{2}\right\} C_{\rho},
$$


so that $f(\lambda)$ diverges at $\lambda=0$. Stationary autoregressive moving average (ARMA) processes satisfy (3.1), and stationary fractionally integrated ARMA (ARFIMA) processes satisfy (3.2), (3.3).

Assumption 1 For $t=0, \pm 1, \ldots$,

$$
\zeta_{t}=\eta_{1 t} g_{t}, \quad \delta_{t}=\nu_{1 t} h_{t}, \quad \varepsilon_{t}=\xi_{1 t} l_{t},
$$

where for real-valued functions $g, h, l$,

$$
g_{t}=g\left(\eta_{2 t}\right), \quad h_{t}=h\left(\nu_{2 t}\right), \quad l_{t}=l\left(\xi_{2 t}\right),
$$

and

(i) $\left\{\eta_{1 t}\right\},\left\{\nu_{1 t}\right\},\left\{\xi_{1 t}\right\}$ are jointly iid processes with zero mean;

(ii) $\left\{\eta_{2 t}\right\}$ is $I(d),\left\{\nu_{2 t}\right\}$ is $I\left(d^{\prime}\right)$, and $\left\{\xi_{2 t}\right\}$ is $I\left(d^{\prime \prime}\right)$, for $d^{\prime} \geq 0, d^{\prime \prime} \geq 0, \max \left\{d^{\prime}, d^{\prime \prime}\right\}<$ $d<1 / 2$;

(iii) $\left\{\eta_{2 t}\right\},\left\{\nu_{2 t}\right\},\left\{\xi_{2 t}\right\}$ are standard Gaussian processes, independent of each other and of $\left\{\eta_{1 t}\right\},\left\{\nu_{1 t}\right\},\left\{\xi_{1 t}\right\}$;

(iv) For some integer $p>1$,

$$
E\left(\eta_{1 t}^{p}\right) E\left\{g^{p}\left(\eta_{2 t}\right) \eta_{2 t}\right\} \neq 0
$$

and for $j=1, \ldots, p-1$,

$$
E\left(\eta_{1 t}^{j} \nu_{1 t}^{p-j}\right) E\left\{g^{j}\left(\eta_{2 t}\right) \eta_{2 t}\right\}=E\left(\eta_{1 t}^{j} \xi_{1 t}^{p-j}\right) E\left\{g^{j}\left(\eta_{2 t}\right) \eta_{2 t}\right\}=0
$$


(v) $\left\{\eta_{1 t}\right\},\left\{\nu_{1 t}\right\},\left\{\xi_{1 t}\right\},\left\{g_{t}\right\},\left\{h_{t}\right\},\left\{l_{t}\right\}$ have finite $4 p$-th moments.

It follows that $\zeta_{t}, \varepsilon_{t}, \delta_{t}$, described by SV models in (3.5), are serially uncorrelated but not serially independent. In particular, $x_{t}^{p}$ is $I(d)$, due to $(3.7)$, which entails $E\left(\eta_{1 t}^{p}\right) \neq 0$ and $g_{t}^{p}-E\left(g_{t}^{p}\right)$ having Hermite rank one. Condition (3.8) ensures a valid cointegrating relationship between $x_{t}^{p}$ and $y_{t}^{p}$, since it implies that the cointegrating error has memory smaller than $d$. If $\eta_{1 t}$ is independent of $\nu_{1 t}, \xi_{1 t}$, the smallest integer satisfying (3.7) will also satisfy (3.8). It is assumed that $p$ is known, which imposes some restrictions on $g$; in practice it may be reasonable to suppose that $p=2$. The most notable exception would occur if $g$ is a symmetric function, e.g. $g_{t}=\left|\eta_{2 t}\right|^{a}, \alpha>0$, but then no finite $p$ satisfies (3.7). This does not rule out a cointegrating relationship of the type that we study below, but the associated conditions would be extremely complex, involving the magnitudes of $d, d^{\prime}, d^{\prime \prime}$, and the Hermite ranks of each centered power of $g_{t}, h_{t}, l_{t}$. Note that for $\gamma \neq 0, g_{t}=\left|\gamma+\eta_{2 t}\right|^{a}$ gives $p=2$. Further discussion concerning the Hermite rank for functional forms in SV models with long memory can be found in Robinson (2001).

An advantage of a low $p$ is that the moment conditions in part (v) of Assumption 1 increase in strength with $p$. Even for $p=2$, the 8-th moment condition that is required seems stringent for most financial data: Jansen and de Vries (1991) and Loretan and Phillips (1994), among others, suggest that several financial time series may have infinite fourth moments. Other parts of Assumption 1 might be relaxed at cost of substantial lengthening of the proof, in particular the mutual independence assumptions of (iii). A consistency result under weaker versions of (iii) could surely be provided with the same theoretical tools, but enumeration of all relevant cross-moments would be a tedious exercise with little added value. The Gaussianity assumption on $\eta_{2 t}, \nu_{2 t}, \xi_{2 t}$ is mitigated by allowing $g, h, l$ to be quite general functions, and without Gaussianity the details 
would be considerably more complex; of course Gaussianity frequently plays a role in short memory SV models also. We do not assume Gaussianity of $\eta_{1 t}, \nu_{1 t}, \xi_{1 t}$.

\section{Consistency of the Narrow Band Least Squares estimate}

We transform (1.1), (1.2) to

$$
Y_{t}=\theta X_{t}+U_{t}
$$

where

$$
\begin{aligned}
& Y_{t}=y_{t}^{p}=\sum_{j=0}^{p}\left(\begin{array}{l}
p \\
j
\end{array}\right) \beta_{1}^{j} \zeta_{t}^{j} \varepsilon_{t}^{p-j}, \quad X_{t}=x_{t}^{p}=\sum_{j=0}^{p}\left(\begin{array}{l}
p \\
j
\end{array}\right) \beta_{2}^{j} \zeta_{t}^{j} \delta_{t}^{p-j}, \quad \theta=\beta^{p}=\frac{\beta_{1}^{p}}{\beta_{2}^{p},} \\
& U_{t}=y_{t}^{p}-\theta x_{t}^{p}=\sum_{j=0}^{p}\left(\begin{array}{c}
p \\
j
\end{array}\right)\left(\beta_{1}^{j} \zeta_{t}^{j} \varepsilon_{t}^{p-j}-\beta^{p} \beta_{2}^{j} \zeta_{t}^{j} \delta_{t}^{p-j}\right)=\sum_{j=0}^{p-1}\left(\begin{array}{c}
p \\
j
\end{array}\right) \beta_{2}^{j} \zeta_{t}^{j}\left(\beta^{j} \varepsilon_{t}^{p-j}-\beta^{p} \delta_{t}^{p-j}\right) .
\end{aligned}
$$

It will follow from (4.1) and Assumption 1 that $Y_{t}$ and $X_{t}$ are cointegrated $I(d)$ processes. As an example, if $p=2$, we have $U_{t}=\varepsilon_{t}^{2}-\beta^{2} \delta_{t}^{2}+2 \beta_{2} \zeta_{t}\left(\beta \varepsilon_{t}-\beta^{2} \delta_{t}\right)$. The memory parameters of $\delta_{t}^{2}, \varepsilon_{t}^{2}$ are bounded by $d^{\prime}, d^{\prime \prime}$ respectively, and therefore smaller than $d$ by part (ii) of Assumption 1. Condition (3.8) guarantees that either $g_{t}$ has Hermite rank greater than one, reducing the memory of the last term by virtue of Theorem 1 , or that both $\zeta_{t} \varepsilon_{t}$ and $\zeta_{t} \delta_{t}$ contain a zero mean and serially uncorrelated multiplicative error, and are therefore white noise. By contrast, (3.7) ensures that $g_{t}^{2}$ in $X_{t}$ has Hermite rank one, and thus retains the memory, $d$, of its underlying volatility process.

Given observations $x_{t}, y_{t}, t=1, \ldots, n$, the NBLS estimate of Robinson (1994a) for $\theta$ is given by

$$
\hat{\theta}_{m}=\frac{\operatorname{Re}\left\{\hat{F}_{X Y}\left(\lambda_{m}\right)\right\}}{\hat{F}_{X X}\left(\lambda_{m}\right)}, \quad 1 \leq m \leq \frac{n}{2},
$$


where $\lambda_{j}=2 \pi j / n$ are the Fourier frequencies, and for generic scalar sequences $a_{t}, b_{t}$, $t=1, \ldots, n$, we define the discretely averaged (cross-) periodogram

$$
\hat{F}_{a b}\left(\lambda_{m}\right)=\frac{2 \pi}{n} \sum_{j=1}^{m} I_{a b}\left(\lambda_{j}\right),
$$

where $I_{a b}(\lambda)=w_{a}(\lambda) \overline{w_{b}(\lambda)}$ is the (cross-) periodogram, and $w_{a}(\lambda)=\sum_{t=1}^{n} a_{t} e^{i t \lambda} / \sqrt{2 \pi n}$ is the discrete Fourier transform of $a_{1}, \ldots, a_{n}$. We can estimate $\beta$ by $\hat{\beta}_{m}=\hat{\theta}_{m}^{1 / p}$, though only up to an unknown sign when $p$ is even.

For $m=[n / 2]$, where [.] denotes integer part, (4.2) reduces to OLS, but for consistency we require, on the contrary:

Assumption 2 The bandwidth sequence $m=m(n)$ satisfies

$$
\frac{1}{m}+\left(\frac{m}{n}\right)^{\epsilon} \log n \rightarrow 0 \text { as } n \rightarrow \infty
$$

for all $\epsilon>0$.

This assumption is slightly stronger than that of Robinson (1994a,b), namely

$$
\frac{1}{m}+\frac{m}{n} \rightarrow 0 \text { as } n \rightarrow \infty
$$

We need (4.3) over (4.4) only in order to handle powers of $g_{t}, h_{t}, l_{t}$ with particular combinations of memory parameters and Hermite ranks, notably for $d=1 / 4$. This case presents no special problems with the method of proof in Robinson (1994a), and is excluded in Robinson (1994b).

For integers $j \in[1, p-1]$ and $k \in[0, p-1]$, denote the Hermite rank of centered $g^{j}$, 
$h^{p-k}, l^{p-k}$ by $r_{g j}, r_{h k}, r_{l k}$ respectively, and introduce the sets

$$
\begin{aligned}
& S_{g}=\left\{j: \beta^{j} E\left(\eta_{1 t}^{j} \varepsilon_{t}^{p-j}\right) \neq \beta^{p} E\left(\eta_{1 t}^{j} \delta_{t}^{p-j}\right), \quad 0<j<p\right\}, \\
& S_{h}=\left\{k: E\left(\nu_{1 t}^{p-k} \zeta_{t}^{k}\right) \neq 0, \quad 0 \leq k<p\right\}, \\
& S_{l}=\left\{k: E\left(\xi_{1 t}^{p-k} \zeta_{t}^{k}\right) \neq 0, \quad 0 \leq k<p\right\}, \\
& S_{g h}=\left\{j: E\left(\eta_{1 t}^{j} \nu_{1 t}^{p-j}\right) \neq 0, \quad 0<j<p\right\}, \\
& S_{g l}=\left\{j: E\left(\eta_{1 t}^{j} \xi_{1 t}^{p-j}\right) \neq 0, \quad 0<j<p\right\} .
\end{aligned}
$$

Intuitively, $U_{t}$ will be expanded as a sum of terms involving the basic processes described in Assumption 1. This allows us to express the autocovariance function of $U_{t}$ as a linear combination of covariances of powers of $g_{t}, h_{t}, l_{t}$ and products of these covariances. The memory of $U_{t}$ will depend only on those terms associated with a nonzero coefficient, in particular for which the white noise component has nonzero mean. These five sets group the particular exponents for which this occurs: $S_{g}, S_{h}, S_{l}$ for terms including only the covariances of powers of $g_{t}, h_{t}, l_{t}$ respectively, and $S_{g h}, S_{g l}$ for cross-products of said covariances. Note that $U_{t}$ does not contain products of $\varepsilon_{t}$ and $\delta_{t}$, and therefore interactions between $h_{t}$ and $l_{t}$ do not occur. Using the convention that the maximum over an empty set is $-\infty$, the slowest rate of decay corresponding to each source is defined by

$$
\begin{aligned}
d_{g}^{*} & =\max _{j \in S_{g}}\left\{\frac{1}{2}-r_{g j}\left(\frac{1}{2}-d\right)\right\}, \\
d_{h}^{*} & =\max _{k \in S_{h}}\left\{\frac{1}{2}-r_{h k}\left(\frac{1}{2}-d^{\prime}\right)\right\}-1\left(d^{\prime}=0\right), \\
d_{l}^{*} & =\max _{k \in S_{l}}\left\{\frac{1}{2}-r_{l k}\left(\frac{1}{2}-d^{\prime \prime}\right)\right\}-1\left(d^{\prime \prime}=0\right), \\
d_{g h}^{*} & =\max _{j \in S_{g h}}\left\{\frac{1}{2}-r_{g j}\left(\frac{1}{2}-d\right)-r_{h j}\left(\frac{1}{2}-d^{\prime}\right)\right\},
\end{aligned}
$$




$$
d_{g l}^{*}=\max _{j \in S_{g l}}\left\{\frac{1}{2}-r_{g j}\left(\frac{1}{2}-d\right)-r_{l j}\left(\frac{1}{2}-d^{\prime \prime}\right)\right\}
$$

where $1(\cdot)$ throughout denotes the identity function, and the memory of $U_{t}$ will be

$$
d^{*}=\max \left\{d_{g}^{*}, d_{h}^{*}, d_{l}^{*}, d_{g h}^{*}, d_{g l}^{*}\right\}
$$

Theorem 2 Under Assumptions 1 and 2, as $n \rightarrow \infty$

$$
\hat{\theta}_{m}-\theta=O_{p}\left(\left(\frac{m}{n}\right)^{d-d_{u}}\right)
$$

where $d_{u}=d^{*} 1\left(d^{*}>0\right)+\epsilon 1\left(d^{*}=0\right)$, for any $\epsilon>0$.

Proof. As in Section 5.3 of Robinson (1994a),

$$
\left|\hat{\theta}_{m}-\theta\right| \leq\left\{\frac{\hat{F}_{U U}\left(\lambda_{m}\right)}{\hat{F}_{X X}\left(\lambda_{m}\right)}\right\}^{\frac{1}{2}}
$$

By Proposition 2,

$$
\left(\frac{m}{n}\right)^{2 d_{u}-1} \hat{F}_{U U}\left(\lambda_{m}\right)=O_{p}(1)
$$

while by Propositions 1, 3, and Slutsky's Theorem,

$$
\frac{\left(\frac{m}{n}\right)^{1-2 d}}{\hat{F}_{X X}\left(\lambda_{m}\right)} \stackrel{p}{\rightarrow} \frac{1}{C^{*}}<\infty
$$

Since $\epsilon$ is arbitrarily small and $d^{*}<d$, it follows that $\hat{\theta}_{m}$ is consistent for $\theta$. Moreover, when $d^{*}>0$, we can write $d-d_{u}=d-d^{*}$, which is the difference between the integration orders of $X_{t}$ and $U_{t}$, where the rate in (4.11) corresponds to that of Robinson and Marinucci (2003). For some particular combinations of memory parameters and 
Hermite ranks, yielding zeros in (4.5)-(4.9), the autocorrelation function is $O\left(j^{-1}\right)$, and an additional $\log n$ factor arises. When such a process dominates in the expansion of $U_{t},(4.3)$ is required to derive (4.11), justifying the appearance of $\epsilon$ in the above rate of convergence.

\section{$5 \quad$ Finite sample properties}

We now present a Monte Carlo study of finite sample performance. For linear processes, Robinson and Marinucci (2003) reported simulation experiments of NBLS with $I(1)$ observables and $I(0)$ cointegrating errors, while Marinucci and Robinson (2001) explored different cases of $I\left(d_{x}\right)$ nonstationary observables and $I\left(d_{e}\right)$ stationary errors. Bandi and Perron (2006) examined NBLS for the regression between realized and implied volatility, generating the data from a discretised continuous time SV model. We employ 50,000 replications of series of various lengths $n$ generated by (1.1), (1.2), (3.5), (3.6), setting $\beta_{1}=\beta_{2}=1$. All basic processes in (3.5), (3.6) are independent of each other, and standard Gaussian. Processes $\eta_{1 t}, \nu_{1 t}, \xi_{1 t}$ in (3.5) were generated as iid, while for the ones in (3.6) the Davies and Harte (1987) algorithm was used to generate $\eta_{2 t}$ as $\operatorname{ARFIMA}(0, d, 0)$ and $\nu_{2 t}, \xi_{2 t}$ as $\operatorname{ARFIMA}\left(0, d^{\prime}, 0\right)$. In most cases $h$ and $l$ are constant functions, and $\nu_{2 t}, \xi_{2 t}$ are not required. For all functions $g$ considered, $p=2$ satisfies Assumption 1.

We compare the performance of NBLS (4.2) with OLS estimates obtained from squared data,

$$
\tilde{\theta}=\frac{\sum\left(X_{t}-\bar{X}\right)\left(Y_{t}-\bar{Y}\right)}{\sum\left(X_{t}-\bar{X}\right)^{2}} .
$$

Of course, (5.1) is not consistent for $\theta$, but it is a simple estimate that a practitioner might optimistically compute. One can furthermore interpret (5.1) as the full band 


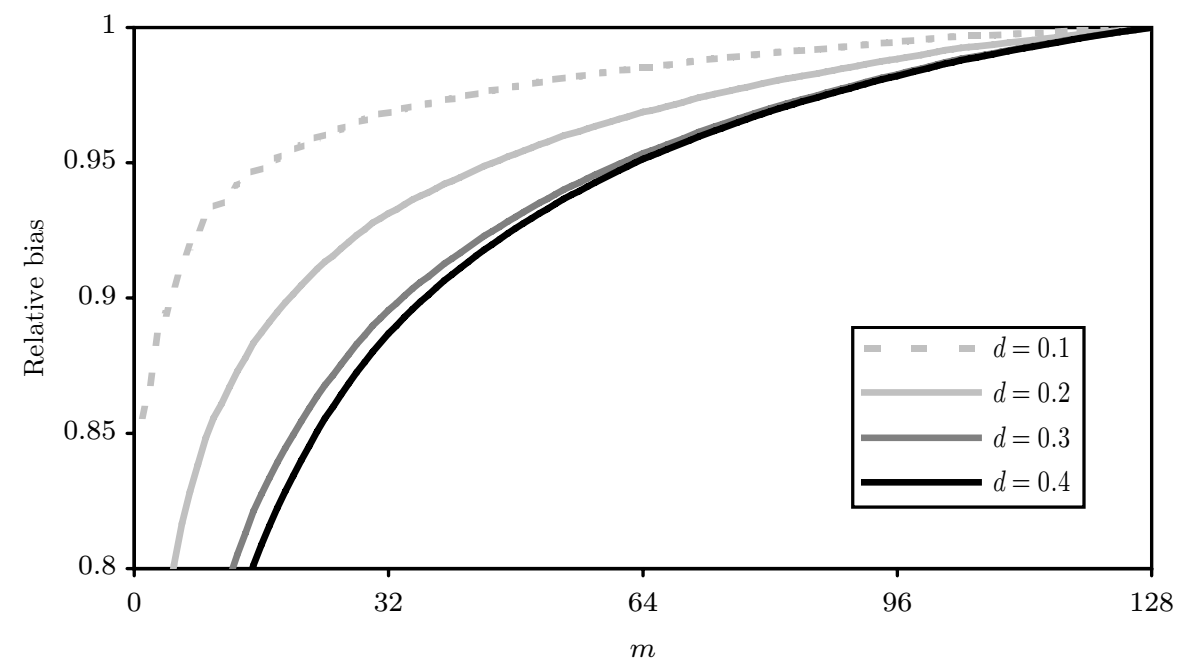

Figure 1: Relative bias of NBLS versus OLS, for varying $m$ and $d$.

version of the proposed NBLS estimate, i.e. (4.2) for $m=[n / 2]$. OLS estimates using the original data, $y_{t}$ on $x_{t}$, performed much worse than both (4.2) and (5.1), and are therefore omitted. We report the bias, standard deviation (SD), and root mean squared error (RMSE) for each estimate. On occasion, relative quantities are reported, meaning the ratio between the corresponding quantity for NBLS and (5.1).

\section{Bandwidth choice}

Theorem 2 highlights the relationship between bandwidth $m$ and rate of convergence. In the first experiment, we present the evolution of relative bias, SD, and RMSE for different $m$ and $d$. We set $n=256, d=0.1,0.2,0.3,0.4, g(x)=\exp (k x)$, with $k$ chosen to satisfy $\operatorname{Var}\left(\zeta_{t}\right)=2$, and $h(x)=l(x)=1$. We chose this value for $\operatorname{Var}\left(\zeta_{t}\right)$ in several experiments in order to balance the contributions of bias and SD to RMSE; the impact of the signal to noise ratio is explored later. 


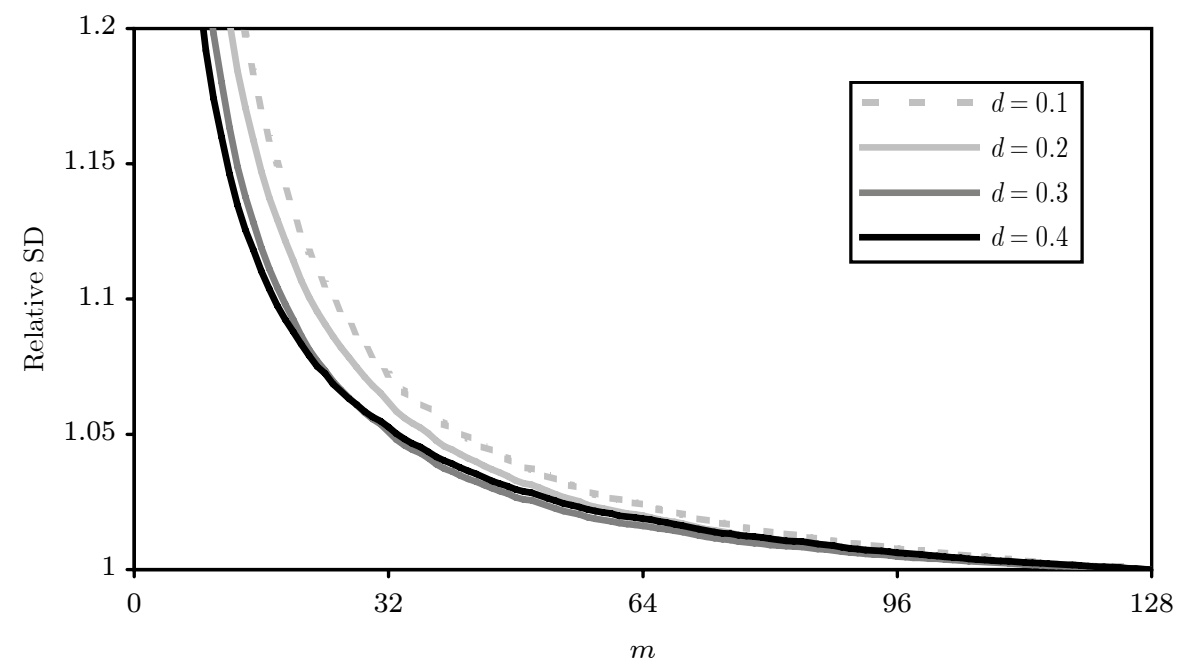

Figure 2: Relative SD of NBLS versus OLS, for varying $m$ and $d$.

Figure 1 shows the bias reduction achieved by NBLS relative to OLS. Not surprisingly, it is greater for small $m$ and large $d$. It is only around frequency zero that the spectral density of $X_{t}$ dominates that of $U_{t}$; frequencies further from the origin are more contaminated by the correlation between $X_{t}$ and $U_{t}$, and contribute more to bias. Also, a higher $d$ indicates a stronger cointegrating relationship, increasing the spectral density of $X_{t}$ around the origin and thus the averaged periodogram.

The increase in SD of NBLS relative to OLS, displayed in Figure 2, is a consequence of discarding high frequency information, and is decreasing in $m$. The influence of $d$ on relative SD appears to be small, especially if compared to Figure 1.

The different profiles of bias and SD give rise to the traditional trade-off in bandwidth choice. Figure 3 presents the relative RMSE of NBLS. For most $m$, NBLS dominates OLS. For this particular $n$, a low $d$ does not provide enough information for NBLS to work, due to the modest bias reductions displayed in Figure 1, making the improvement over OLS negligible. The RMSE is essentially a flat function of $m$, implying that any $m$ 


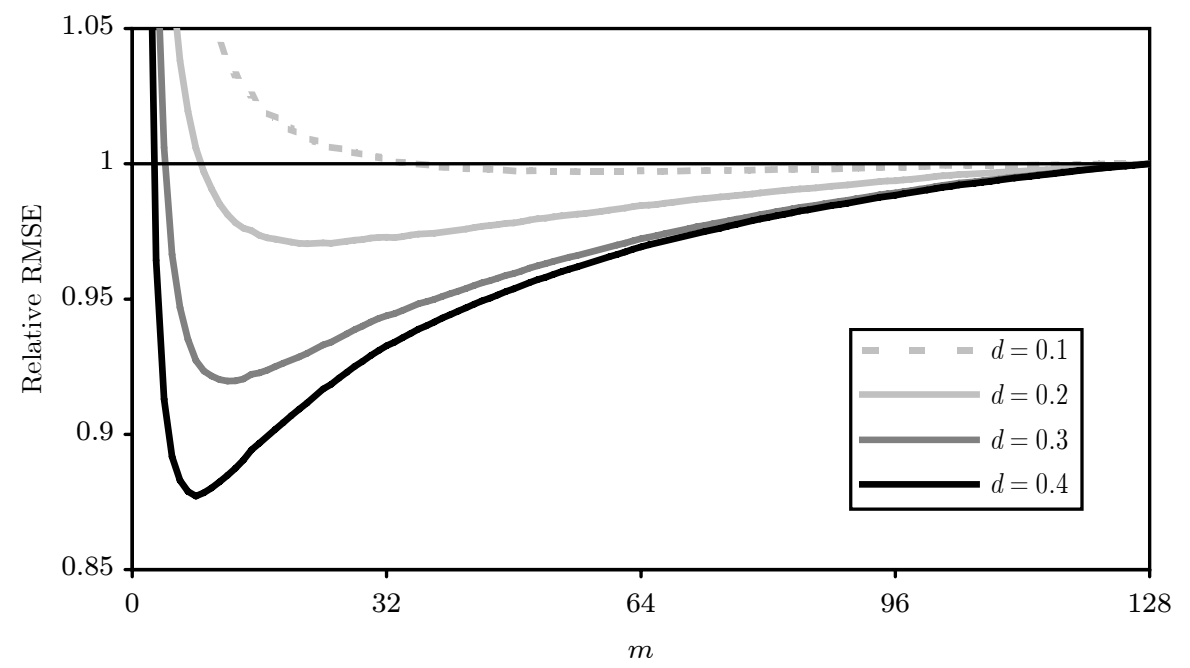

Figure 3: Relative RMSE of NBLS versus OLS, for varying $m$ and $d$.

above a certain threshold, thereby taking in OLS, attains similar RMSE. However, note that an increase in $n$ should have a similar effect to an increase in $d$ on RMSE, although it will be minimized at a different $m$. This effect is explored in the next subsection. Higher $d$ lead to very low values for the optimal $m$, and more significant improvements in RMSE. For $d=0.4$, a noticeable reduction is already achieved, of over $10 \%$ for a number of different $m$. It should also be noted that if the bandwidth selection is larger than optimal, it is still possible to considerably reduce RMSE, while choosing too small an $m$ can lead to an undesirably large SD.

\section{Memory in signal}

We now investigate the influence of $n$ and $d$ on the performance of the estimates. We consider $n=256,512,1024,2048$ and $d=0.1,0.2,0.3,0.4$. As before, $g(x)=\exp (k x)$, with $k$ chosen to satisfy $\operatorname{Var}\left(\zeta_{t}\right)=2$, and $h(x)=l(x)=1$. In this experiment and in the 
following ones, we evaluate NBLS at the bandwidth $m^{*}$ that minimizes RMSE. Although this is not a feasible choice in the usual sense, it gives an indication of potential gains. We also present results for the feasible bandwidth rule $m=\left[n^{0.5}\right]$, often used in practical applications. Table 1 summarizes the results.

As expected, the RMSE of all estimates improves with $n$. For even moderate $n$, NBLS has the lowest RMSE, being less biased than OLS; while OLS attains a lower SD, especially for small $n$, its larger bias makes it worse. This gain in RMSE seems negligible for low $d$, as suggested by Figure 3, but becomes noticeable for higher $d$.

Both bias and SD of OLS increase with $d$. Both also decrease with $n$, but while SD seems to be rapidly converging to zero, bias decreases rather slowly and appears to stabilise at some substantial non-zero value. The effect of $d$ on NBLS bias is ambiguous for small $n$, but as $n$ increases bias becomes clearly decreasing with $d$. In most cases, SD increases with $d$ for NBLS, but to a much smaller extent than for OLS.

The results for $m=\left[n^{0.5}\right]$ and $m^{*}$ are comparable for intermediate $d$. For low $d$, the feasible rule underestimates the optimal bandwidth, and as a result the increase in variance does not compensate for the modest gains in bias reduction. By contrast, the optimal bandwidth is greatly overestimated for large $d$, not taking advantage of the sizeable bias reduction potential. Even so, the feasible rule is superior to OLS in all but one case, and its RMSE is often much closer to the optimal than to the full band one.

The bias reduction of NBLS becomes quite large with $n$, while the variance penalty is always of small magnitude. In fact, for large $n$ and $d$, NBLS actually dominates OLS in both SD and bias. The improvement in performance for high $d$ and the rate of decay of RMSE seem compatible with the asymptotic result of Theorem 2.

While Figure 3 and Table 1 both illustrate the high sensitivity of $m^{*}$ to $d$, caused by the different scope for bias reduction in each case, $m^{*}$ does not appear to grow 


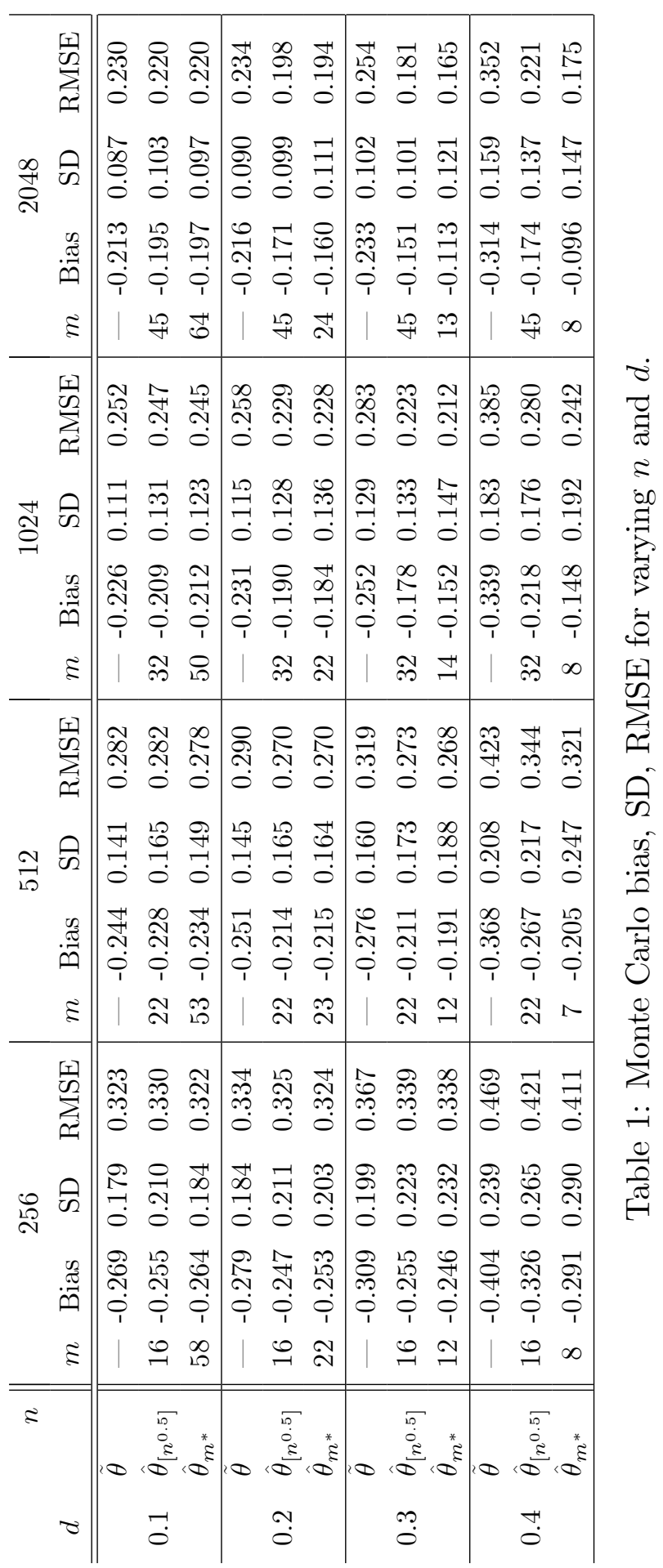


with $n$. This is surely a small sample effect, as NBLS is only consistent if $m \rightarrow \infty$. As a consequence, $m^{*}$ will diverge when bias becomes negligible compared to SD, a situation which does not occur in the sample sizes considered. Since Theorem 2 shows that convergence of $\hat{\theta}_{m}$ is faster the slower $m$ grows, this phenomenon is not entirely surprising.

\section{Memory in signal and noise}

In Table $2, d$ is kept constant, while we introduce long memory in the errors. We set $g(x)=\exp \left(k_{1} x\right)$ and $h(x)=l(x)=\exp \left(k_{2} x\right)$, with $k_{1}, k_{2}$ chosen to satisfy $\operatorname{Var}\left(\zeta_{t}\right)=10$ and $\operatorname{Var}\left(\delta_{t}\right)=\operatorname{Var}\left(\varepsilon_{t}\right)=2$. These values were again chosen to balance contributions of bias and SD to RMSE. We consider $n=256,512,1024,2048, d=0.4$ and $d^{\prime}=0,0.1$, $0.2,0.3$.

The results are very similar to the previous experiment, but here $d-d^{\prime}$ takes the role of $d$. As before, RMSE improves with $n$ for all estimates. OLS displays similar patterns of bias and SD across $d-d^{\prime}$ and $n$, with the exception that SD decays much more slowly with $n$. The bias of NBLS decreases with $d-d^{\prime}$ for all $n$; for $n>256$ even $\mathrm{SD}$ decreases with $d-d^{\prime}$. A surprising fact in this case is related to the variance/bias trade-off of NBLS. While this can be found in small samples, as $n$ increases it starts dominating OLS in both bias and variance. The evolution of $m^{*}$ is also similar to the previous section.

We do not directly address the impact of short-run dynamics in finite samples, as we expect its consequences to be qualitatively analogous to the linear case. As reported in Robinson and Marinucci (2003), the presence of short memory positive autocorrelation in the common factor should boost the spectral peak in small samples, reducing bias in a similar manner to a higher $d$ in Table 1 ; conversely, negative autocorrelation in 


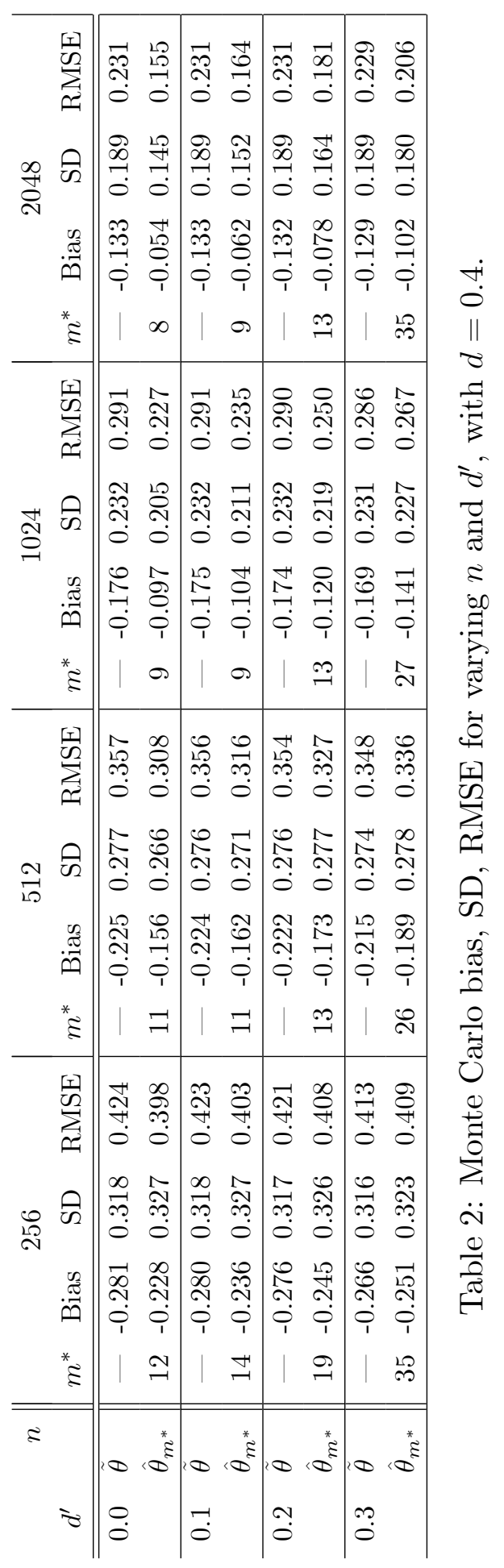


the common factor should have a similar effect to a lower $d$. The impact of short-run dynamics in the idiosyncratic components would predictably be the opposite: positive correlation should worsen performance similarly to an increase in $d^{\prime}$ in Table 2, while negative correlation would be associated with a dampened $d^{\prime}$. Both effects should become negligible as $n$ grows.

\section{Signal to noise ratio}

This experiment investigates the influence of the signal to noise $(\mathrm{S} 2 \mathrm{~N})$ ratio on the performance of NBLS. We use $g(x)=\exp (k x)$, such that $\operatorname{Var}\left(\zeta_{t}\right)=2$, and $h(x)=$ $l(x)=\sigma$, so that $\operatorname{Var}\left(\delta_{t}\right)=\operatorname{Var}\left(\varepsilon_{t}\right)=\sigma^{2}$, for $\sigma^{2}=0.25,0.5,1,2,4$. The results obtained for different $d$ were qualitatively similar, so we report results only for $d=0.3$ and $n=256,512,1024,2048$. Since it is unreasonable to compare absolute performance for different $\mathrm{S} 2 \mathrm{~N}$ ratios, in Table 3 we focus on relative performance only. We also report the ratio between bias and SD. Although we refer to $\operatorname{Var}\left(\zeta_{t}\right) / \operatorname{Var}\left(\delta_{t}\right)$ as the S2N ratio, for simplicity, it is only an accurate description for the regression in levels. For $x_{t}^{2}=\zeta_{t}^{2}+2 \zeta_{t} \delta_{t}+\delta_{t}^{2}$, the dominant term is $\zeta_{t}^{2}$, and even there $\eta_{1 t}^{2}$ could be considered a multiplicative noise. Hence, the definition of the "true" S2N ratio would be ambiguous, but it would be arguably smaller than the one in levels.

NBLS performs best when bias and SD are balanced. The regressor $X_{t}$ consists of two parts: a long memory component containing a dominating pole at frequency zero, and a component with less memory not orthogonal to $U_{t}$. In this case, it is actually short memory, since $\delta_{t}$ is iid. If the $\mathrm{S} 2 \mathrm{~N}$ ratio is very large, the first component will dominate the second even at frequencies distant from zero. As a result, any large enough $m$ will perform well, and even with OLS, bias will contribute very little to RMSE and gains from NBLS will be small. On the other hand, for very small S2N, the second component 


\begin{tabular}{|c|c|c|c|c|c|c|c|c|c|c|c|c|}
\hline$n$ & \multicolumn{3}{|c|}{256} & \multicolumn{3}{|c|}{512} & \multicolumn{3}{|c|}{1024} & \multicolumn{3}{|c|}{2048} \\
\hline S2N & Bias & SD & RMSE & Bias & SD & RMSE & Bias & SD & RMSE & Bias & SD & RMSE \\
\hline 0.5 & 0.994 & 1.280 & 0.998 & 0.981 & 1.960 & 0.992 & 0.961 & 2.924 & 0.980 & 0.927 & 4.141 & 0.956 \\
\hline 1 & 910 & .406 & 0.95 & & 1.682 & 0.902 & & 1.773 & 0 & 21 & 1.955 & .731 \\
\hline 2 & 0.797 & 1.163 & 0.920 & 691 & 1.175 & 0.839 & .604 & 1.144 & 0.749 & 0.485 & 1.181 & 0.650 \\
\hline 4 & 34 & 050 & 0 & & 1.057 & & 2 & 1.048 & & 57 & 1.059 & 31 \\
\hline \multirow[t]{2}{*}{8} & 6 & 1.000 & 1.000 & 9 & 1.013 & 0.989 & 73 & 1.019 & 0.965 & 0.683 & 1.016 & 0.922 \\
\hline & \multicolumn{3}{|c|}{ Bias / SD } & \multicolumn{3}{|c|}{ Bias / SD } & \multicolumn{3}{|c|}{ Bias / SD } & \multicolumn{3}{|c|}{ Bias / SD } \\
\hline $\mathrm{S} 2 \mathrm{~N}$ & $m^{*}$ & OLS & NBLS & $m^{*}$ & OLS & NBLS & $m^{*}$ & OLS & NBLS & $m^{*}$ & OLS & NBLS \\
\hline 0.5 & 52 & -9.29 & -7.22 & 27 & -11.42 & -5.72 & 17 & -14.19 & -4. & 12 & -17.26 & -3.86 \\
\hline 1 & 12 & -3.38 & -2.1 & . & -3.72 & -1.81 & 8 & -4.14 & -1.72 & 7 & -4.69 & -1.49 \\
\hline 2 & 12 & -1.55 & -1.06 & 12 & -1.73 & -1.02 & 14 & -1.95 & -1.03 & 13 & -2.28 & -0.94 \\
\hline 4 & 26 & -0.82 & -0.65 & 28 & -0.93 & -0.65 & 33 & -1.09 & -0.68 & 35 & -1.31 & -0.69 \\
\hline 8 & 121 & -0.43 & -0.43 & 87 & -0.49 & -0.42 & 86 & -0.57 & -0.43 & 90 & -0.69 & -0.47 \\
\hline
\end{tabular}

Table 3: Monte Carlo relative bias, SD, RMSE of NBLS versus OLS, for varying $n$ and $\mathrm{S} 2 \mathrm{~N}$, with $d=0.3$.

will be relatively large, dominating the signal even at frequencies close to zero. In small samples, an attempt to reduce bias by only choosing informative frequencies would imply the use of very small $m$, which would force SD to be too high (see Figure 2). In this case, NBLS would also provide little gains, as the cost (in terms of SD) of reducing bias is too high for RMSE.

With OLS the ratio between bias and SD increases with $n$. This is expected, since OLS still converges in probability to a constant. In NBLS, the ratio is very close to that of OLS in small samples. From that point, it increases with $n$ if it was originally small, but decreases if it was originally large. It appears that this ratio will stabilize at some value close to unity for large enough $n$, and from that point on NBLS will have a noticeable RMSE improvement over OLS. 


\section{Nonlinearity}

To investigate the influence of nonlinearity on NBLS, Table 4 reports its performance in three different settings, for $n=256,512,1024,2048$ and $d=0.1,0.2,0.3,0.4$. The nonlinear setting (NL), already used in the first two subsections, has $g(x)=\exp (k x)$, with $k$ chosen to satisfy $\operatorname{Var}\left(\zeta_{t}\right)=2$, and $h(x)=l(x)=1$. In the other two we deviate from (1.1), (1.2), (3.5), (3.6), using instead $Y_{t}=X_{t}+u_{t}, X_{t}=f_{t}+v_{t}$, where $u_{t}, v_{t}$ are generated as iid mean zero Gaussian with $\operatorname{Var}\left(u_{t}\right)=20, \operatorname{Var}\left(v_{t}\right)=6$, and $\operatorname{Cov}\left(u_{t}, v_{t}\right)=-10$. In a fully linear setting $(\mathrm{L})$, we generate $f_{t}$ as a Gaussian mean zero $\operatorname{ARFIMA}(0, d, 0)$, with $\operatorname{Var}\left(f_{t}\right)=44$. In a linear setting with a multiplicative noise $(\mathrm{MN})$, we set $f_{t}=\eta_{1 t}^{2} z_{t}$, where $\eta_{1 t}$ is iid standard Gaussian while $z_{t}$ is independently generated as a Gaussian $\operatorname{ARFIMA}(0, d, 0)$, with $E\left(z_{t}\right)=2$ and $\operatorname{Var}\left(z_{t}\right)=12$. The chosen moments replicate those of corresponding processes in the nonlinear setting.

Both OLS and NBLS perform much better under L than NL, while performance under MN falls in the middle. A similar ordering is found in relative performance (not shown), since a relatively stable, large bias of OLS estimates throughout makes variations in RMSE smaller than for NBLS. Although some of the gap in performance should be a consequence of nonlinearity, significant excess kurtosis in NL and MN is arguably the dominant factor, since it directly affects the variance of the periodogram. In MN, the kurtosis of $f_{t}$ is around 77, while in NL it is around 3523 for $f_{t}, 36$ for $v_{t}$, and 30 for $u_{t}$.

\section{Volatility function}

Finally, we explore the impact of the functional form of the volatility function $g$. considering $g(x)=\exp (k x),(1+k x)^{2},|1+k x|$, with $k$ chosen in each case so that $\operatorname{Var}\left(\zeta_{t}\right)=2$. We set $h(x)=l(x)=1$ and $d=0.1,0.2,0.3,0.4$. Table 5 presents the results for $n=512$, where the properties of each estimate seem robust to the choice 


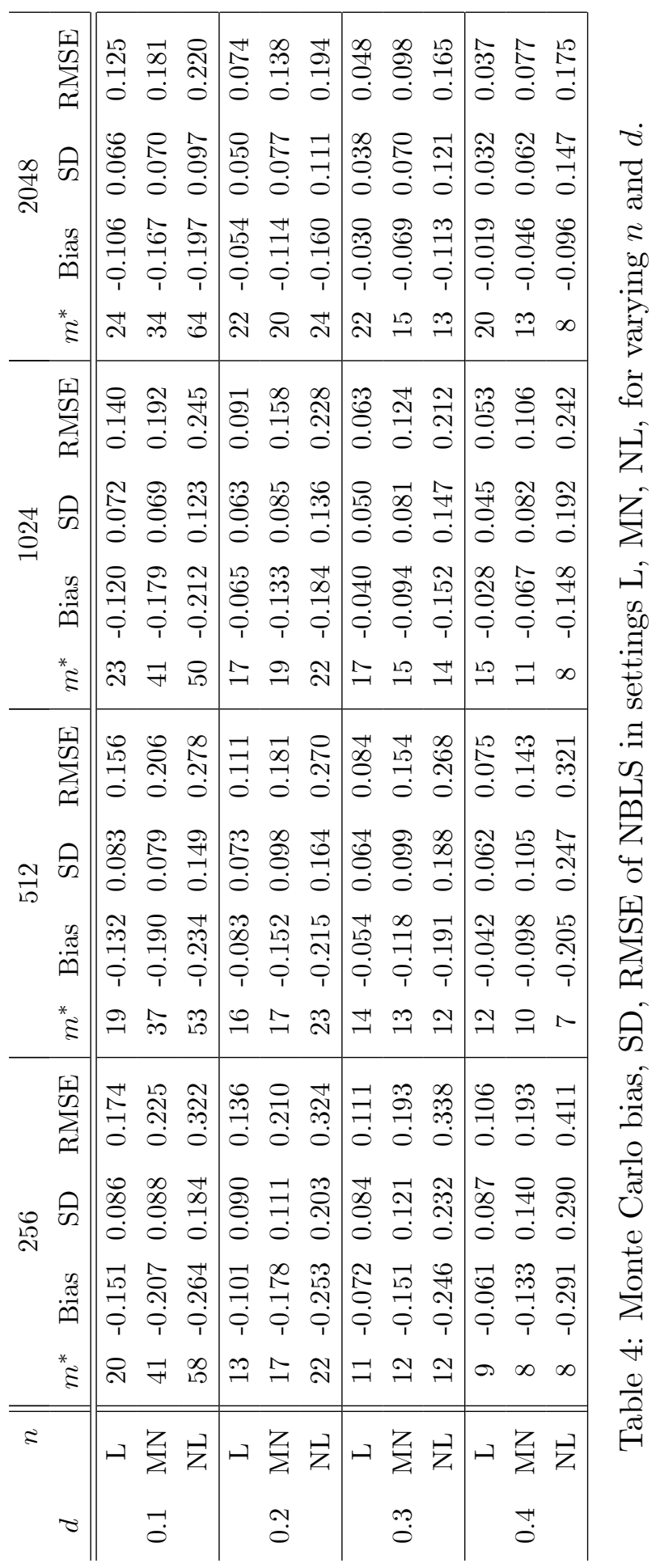




\begin{tabular}{|cl||cccc|cccc|cccc|}
\multicolumn{1}{l||}{} & \multicolumn{6}{c||}{$g$} & \multicolumn{6}{c|}{$\exp (x)$} & \multicolumn{4}{c|}{$(1+x)^{2}$} & \multicolumn{4}{|c|}{$|1+x|$} \\
$d$ & & $m^{*}$ & Bias & SD & RMSE & $m^{*}$ & Bias & SD & RMSE & $m^{*}$ & Bias & SD & RMSE \\
\hline \hline 0.1 & $\tilde{\theta}$ & - & -0.244 & 0.141 & 0.282 & - & -0.262 & 0.122 & 0.289 & - & -0.291 & 0.112 & 0.312 \\
& $\hat{\theta}_{m^{*}}$ & 53 & -0.234 & 0.149 & 0.278 & 47 & -0.250 & 0.136 & 0.284 & 52 & -0.280 & 0.128 & 0.308 \\
\hline 0.2 & $\tilde{\theta}$ & - & -0.251 & 0.145 & 0.290 & - & -0.267 & 0.125 & 0.295 & - & -0.295 & 0.114 & 0.316 \\
& $\hat{\theta}_{m^{*}}$ & 23 & -0.215 & 0.164 & 0.270 & 17 & -0.219 & 0.159 & 0.271 & 23 & -0.255 & 0.148 & 0.295 \\
\hline 0.3 & $\tilde{\theta}$ & - & -0.276 & 0.160 & 0.319 & - & -0.285 & 0.136 & 0.316 & - & -0.308 & 0.122 & 0.332 \\
& $\hat{\theta}_{m^{*}}$ & 12 & -0.191 & 0.188 & 0.268 & 12 & -0.190 & 0.174 & 0.258 & 13 & -0.220 & 0.174 & 0.280 \\
\hline 0.4 & $\tilde{\theta}$ & - & -0.368 & 0.208 & 0.423 & - & -0.358 & 0.181 & 0.401 & - & -0.359 & 0.150 & 0.390 \\
& $\hat{\theta}_{m^{*}}$ & 7 & -0.205 & 0.247 & 0.321 & 8 & -0.190 & 0.219 & 0.290 & 8 & -0.205 & 0.219 & 0.299 \\
\hline
\end{tabular}

Table 5: Monte Carlo bias, SD, RMSE for varying $g(x)$ and $d$, with $n=512$.

of volatility function. Normalizing $\operatorname{Var}\left(\zeta_{t}\right)$ appears to be sufficient to capture most of the differences across functions. Results for other $n$ are similar and available from the authors upon request.

\section{Final comments}

To our knowledge this paper represents the first treatment of fractional cointegration in the context of nonlinear processes. The stationary environment, the SV models employed, and the NBLS estimate seem well motivated by applications in finance. Our model is semiparametric both in the sense that only assumptions about low frequency behavior are required, and the volatility functions are nonparametric. While the nonlinear setting necessitates a considerably more complex proof of consistency of NBLS than earlier ones, a comparable result is obtained, with rate of convergence depending essentially on the strength of the cointegrating relation, namely the gap between integration orders of observables and cointegrating error. Monte Carlo results show encouraging performances in moderate sample sizes across a variety of specifications.

As always, consistency results are reassuring only in very large data sets. Though 
these do exist in finance, one would like a limit distributional result that could be used in statistical inference. Christensen and Nielsen (2006) have achieved this in a simpler setting, indeed with regressor and disturbance assumed incoherent at frequency zero, and linear process (in conditionally homoscedastic md innovations) assumptions. In general, not only is the proof likely to be much more complicated than even our proof of Theorem 2 , but the limit distribution is likely to be non-standard for various combinations of memory parameters, though a bootstrap procedure might be investigated. By analogy with experience in $I(1) / I(0)$ cointegrated models (e.g. Johansen, 1991; Phillips, 1991), it may be possible to obtain estimates with nicer asymptotic distributional properties, in particular leading to Wald statistics with null limiting $\chi^{2}$ distributions. However, in our nonlinear setting it is not immediately obvious that the sort of transformations used in those references to achieve the necessary "whitening" will be successful, the estimates would require preliminary estimation of memory parameters, and proofs would be significantly more complicated. Nevertheless, those wishing to embark on limit distributional proofs for NBLS or other estimates in our SV setting should find techniques described in the present paper relevant.

Though our Monte Carlo study addressed the choice of bandwidth $m$, it would evidently be desirable to develop a feasible rule for bandwidth selection. In a Gaussian or linear setting, Robinson (1994b) developed formulae for minimum-MSE bandwidth with respect to the basic averaged periodogram statistic, and these were further analyzed by Delgado and Robinson (1996). In principle these could be extended to the NBLS estimate, though the formulae will be highly complex, and feasible versions would require estimating memory parameters and other quantities. As in other circumstances, sensitivity to choice of $m$ can be assessed by a "window-closing" approach, computing NBLS over a sensibly chosen grid of $m$ values; since discrete Fourier transforms at all 
Fourier frequencies can be obtained simultaneously by the Fast Fourier Transform, and NBLS is algebraically simple, this can cheaply be achieved, indeed a simple recursion deals with unit or other increases in $m$.

The bulk of the fractional and non-fractional cointegration literature assumes nonstationary observables. The motivation usually comes from macroeconomics, but nonstationarity can often appear in financial time series also. The modelling of nonstationary series via analogues of (4.1) is itself a somewhat open topic, but given that $X_{t}$ has a kind of $I(d)$ property, for $d \geq 1 / 2$, some of the arguments of Robinson and Marinucci (2001) should be relevant in establishing rates of convergence of NBLS. Indeed, these authors, following Stock (1987) in the $I(1) / I(0)$ case, found OLS also to be consistent here, though in some circumstances NBLS has bias of smaller order. The nonstationary $X_{t}$ case is in some respects technically easier than the stationary one, because consistency of OLS follows from the domination of sums of squares of $U_{t}$ by those of $X_{t}$.

Other directions of research could extend (1.1), (1.2) to more than two observables, and then possibly to allow more than one common factor, i.e. more than one cointegrating relation. On the one hand, cointegrating relations between a potentially large number of asset returns can be of interest, while on the other, Ross (1976) and others suggest the need for additional unobservable factors in asset pricing models. It should be possible to determine a form of multivariate regression linking the observables, analogous to (4.1), and then a multivariate extension for NBLS (4.2). Its consistency, subject to identifiability conditions, can then be established under an analogue of Assumption 1, using Theorem 1 and techniques employed in the proof of Theorem 2, though of course the details would be even more complicated. The issue of determining cointegrating rank, and thus the number of common factors, is of more pressing concern than in our simple model (1.1), (1.2), but procedures such as those of Robinson and Yajima (2002) 
might be employed in practice. Again, their theoretical justification in our setting would require considerable further work.

\section{Acknowledgements}

Both authors' research was supported by ESRC Grant R000239936. Gonçalves da Silva's research was also supported by FCT Grant SFRH/BD/4783/2001, and Robinson's research was also supported by ESRC Grant RES-062-23-0036. We thank the co-editor and two anonymous referees for their valuable comments, and Greg Connor and Dennis Kristensen for helpful comments on previous versions of the paper.

\section{References}

Andersen, T.G. \& T. Bollerslev (1997) Intraday periodicity and volatility persistence in financial markets. Journal of Empirical Finance 4, 115-158.

Baillie, R.T., T. Bollerslev \& H.O. Mikkelsen (1996) Fractionally integrated generalized autoregressive conditional heteroskedasticity. Journal of Econometrics 74, 3-30.

Bandi, F.M. \& B. Perron (2006) Long memory and the relation between implied and realized volatility. Journal of Financial Econometrics 4, 636-670.

Bollerslev, T. (1986) Generalized autoregressive conditional heteroskedasticity. Journal of Econometrics $31,302-327$.

Bollerslev, T. \& H.O. Mikkelsen (1996) Modeling and pricing long memory in stock market volatility. Journal of Econometrics 73, 151-184.

Breidt, F.J., N. Crato \& P. de Lima (1998) The detection and estimation of long memory in stochastic volatility. Journal of Econometrics 83, 325-348.

Brunetti, C. \& C.L. Gilbert (2000) Bivariate FIGARCH and fractional cointegration. Journal of Empirical Finance 7, 509-530. 
Christensen, B.J. \& M.Ø. Nielsen (2006) Asymptotic normality of narrow-band least squares in the stationary fractional cointegration model and volatility forecasting. Journal of Econometrics 133, 343-371.

Davies, R.B. \& D.S. Harte (1987) Tests for Hurst effect. Biometrika 74, 95-101.

Delgado, M.A. \& P.M. Robinson (1996) Optimal spectral bandwidth for long memory. Statistica Sinica $6,97-112$.

Ding, Z. \& C.W.J. Granger (1996) Modeling volatility persistence of speculative returns: A new approach. Journal of Econometrics 73, 185-215.

Ding, Z., C.W.J. Granger \& R.F. Engle (1993) A long memory property of stock market returns and a new model. Journal of Empirical Finance 1, 83-106.

Engle, R.F. (1982) Autoregressive conditional heteroscedasticity with estimates of the variance of United Kingdom inflation. Econometrica 50, 987-1008.

Engle, R.F. \& T. Bollerslev (1986) Modelling the persistence of conditional variances. Econometric Reviews 5, 1-50, 81-87.

Harvey, A.C. (1998) Long memory in stochastic volatility. In J.L. Knight \& S.E. Satchell (eds), Forecasting volatility in financial markets, pp. 307-320. Quantitative Finance Series, ButterworthHeinemann.

Hualde, J. \& P.M. Robinson (2006) Semiparametric estimation of fractional cointegration. STICERD Discussion Paper EM/2006/502.

Hurvich, C.M., E. Moulines \& P. Soulier (2005) Estimating long memory in volatility. Econometrica $73,1283-1328$.

Jansen, D.W. \& C.G. de Vries (1991) On the frequency of large stock returns: putting booms and busts into perspective. Review of Economics and Statistics 73, 18-24.

Johansen, S. (1991) Estimation and hypothesis testing of cointegration vectors in Gaussian vector autoregressive models. Econometrica 59, 1551-1580.

Loretan, M. \& P.C.B. Phillips (1994) Testing the covariance stationarity of heavy-tailed time series. Journal of Empirical Finance 1, 211-248. 
Marinucci, D. \& P.M. Robinson (2001) Semiparametric fractional cointegration analysis. Journal of Econometrics 105, 225-247.

Merton, R.C. (1973) An intertemporal capital asset pricing model. Econometrica 41, 867-887.

Phillips, P.C.B. (1991) Optimal inference in cointegrated systems. Econometrica 59, 283-306.

Robinson, P.M. (1991) Testing for strong serial correlation and dynamic conditional heteroskedasticity in multiple regression. Journal of Econometrics 47, 67-84.

Robinson, P.M. (1994a) Semiparametric analysis of long-memory time series. Annals of Statistics 22, 515-539.

Robinson, P.M. (1994b) Rates of convergence and optimal spectral bandwidth for long range dependence. Probability Theory and Related Fields 99, 443-473.

Robinson, P.M. (2001) The memory of stochastic volatility models. Journal of Econometrics 101, 195-218.

Robinson, P.M. \& D. Marinucci (2001) Narrow band analysis of nonstationary processes. Annals of Statistics 29, 947-986.

Robinson, P.M. \& D. Marinucci (2003) Semiparametric frequency domain analysis of fractional cointegration. In P.M. Robinson (ed.), Time series with long memory, pp. 334-373. Advanced Texts in Econometrics, Oxford University Press.

Robinson, P.M. \& Y. Yajima (2002) Determination of cointegrating rank in fractional systems. Journal of Econometrics 106, 217-241.

Ross, S.A. (1976) The arbitrage theory of capital asset pricing. Journal of Economic Theory 13, 341-360.

Sharpe, W.F. (1964) Capital asset prices: A theory of market equilibrium under conditions of risk. Journal of Finance 19, 425-442.

Slepian, D. (1972) On the symmetrized Kronecker power of a matrix and extensions of Mehler's formula for Hermite polynomials. SIAM Journal on Mathematical Analysis 3, 606-616.

Stock, J.H. (1987) Asymptotic properties of least squares estimators of cointegrating vectors. Econometrica 55, 1035-1056.

Taylor, S.J. (1986) Modelling financial time series. John Wiley and Sons. 
Whistler, D.E.N. (1990) Semiparametric models of daily and intradaily exchange rate volatility. $\mathrm{PhD}$ thesis, University of London.

Yong, C.H. (1974) Asymptotic behaviour of trigonometric series. Chinese University of Hong Kong.

Zygmund, A. (1977) Trigonometric series. Cambridge University Press.

\section{Appendix A: Proof of Theorem 1}

Throughout the proof, we denote $P=P_{J}, Q=Q_{J}$, and $R_{j}=R_{J, j}, j \in P$. Furthermore, all sums and products run over $P$ unless otherwise stated. We have

$$
E\left(\prod_{j} f_{j}\right)=\int_{\mathbb{R}^{J}} \prod_{j} f_{j} \phi_{J}(\mu ; \Omega) \mathrm{d} \mu,
$$

where $\phi_{J}(\mu ; \Omega)$ denotes the density function of $\mu=\left(\mu_{1}, \ldots, \mu_{J}\right)^{\prime}$ and $\Omega=E\left(\mu \mu^{\prime}\right)$. From (22) of Slepian (1972) and $(2.1), \phi_{J}(\mu ; \Omega)$ is

$$
\begin{gathered}
\sum_{v_{\alpha}=0: \alpha \in Q}^{\infty} \prod_{\alpha \in Q} \frac{\rho_{\alpha}^{v_{\alpha}}}{v_{\alpha} !} \prod_{j}\left\{\left(\frac{\partial}{\partial \mu_{j}}\right)^{w_{j}} \phi\left(\mu_{j}\right)\right\}=\sum_{v_{\alpha}=0: \alpha \in Q}^{\infty} \prod_{\alpha \in Q} \frac{\rho_{\alpha}^{v_{\alpha}}}{v_{\alpha} !} \prod_{j}\left\{(-1)^{w_{j}} H_{w_{j}}\left(\mu_{j}\right) \phi\left(\mu_{j}\right)\right\} \\
=\sum_{v_{\alpha}=0: \alpha \in Q}^{\infty} \prod_{\alpha \in Q} \frac{\rho_{\alpha}^{v_{\alpha}}}{v_{\alpha} !} \prod_{j}\left\{H_{w_{j}}\left(\mu_{j}\right) \phi\left(\mu_{j}\right)\right\}
\end{gathered}
$$

since $\sum_{j} w_{j}=2 \sum_{\alpha \in Q} v_{\alpha}$ is even. Using (A.2) in (A.1), $E\left(\prod_{j} f_{j}\right)$ is

$$
\begin{aligned}
& \int_{\mathbb{R}^{J}} \prod_{j} f_{j}\left(\mu_{j}\right) \sum_{v_{\alpha}=0: \alpha \in Q}^{\infty} \prod_{\alpha \in Q} \frac{\rho_{\alpha}^{v_{\alpha}}}{v_{\alpha} !} \prod_{j}\left\{H_{w_{j}}\left(\mu_{j}\right) \phi\left(\mu_{j}\right)\right\} \mathrm{d} \mu \\
& =\sum_{v_{\alpha}=0: \alpha \in Q}^{\infty} \prod_{\alpha \in Q} \frac{\rho_{\alpha}^{v_{\alpha}}}{v_{\alpha} !} \int_{\mathbb{R}^{J}} \prod_{j}\left\{f_{j}\left(\mu_{j}\right) H_{w_{j}}\left(\mu_{j}\right) \phi\left(\mu_{j}\right)\right\} \mathrm{d} \mu \\
& =\sum_{v_{\alpha}=0: \alpha \in Q}^{\infty} \prod_{\alpha \in Q} \frac{\rho_{\alpha}^{v_{\alpha}}}{v_{\alpha} !} \prod_{j} E\left\{f_{j}\left(\mu_{j}\right) H_{w_{j}}\left(\mu_{j}\right)\right\}=\sum_{v_{\alpha}=0: \alpha \in Q}^{\infty} \prod_{j} G_{j, w_{j}} \prod_{\alpha \in Q} \frac{\rho_{\alpha}^{v_{\alpha}}}{v_{\alpha} !} .
\end{aligned}
$$


This proves (2.3). For the remainder of the proof, we use the Cauchy-Schwarz inequality in

$$
\begin{aligned}
\left|a_{q}\right| & \leq \sum_{\substack{v_{\alpha} \geq 0: \\
\Sigma v_{\alpha}=q, \alpha \in Q}}\left|\prod_{j} G_{j, w_{j}} \prod_{\alpha \in Q} \frac{\rho_{\alpha}^{v_{\alpha}}}{v_{\alpha} !}\right| \\
& \leq \sum_{\substack{v_{\alpha} \geq 0: \\
\Sigma v_{\alpha}=q, \alpha \in Q}} \prod_{j} \frac{\left|G_{j, w_{j}}\right|}{\sqrt{w_{j} !}} \prod_{j} \sqrt{w_{j} !} \prod_{\alpha \in Q} \frac{\left|\rho_{\alpha}\right|^{v_{\alpha}}}{v_{\alpha} !} \leq\left(A_{q} B_{q}\right)^{\frac{1}{2}},
\end{aligned}
$$

where

$$
A_{q}=\sum_{\substack{v_{\alpha} \geq 0: \\ v_{\alpha}=q, \alpha \in Q \\ w_{j} \geq r_{j}, j \in P}} \prod_{j} \frac{G_{j, w_{j}}^{2}}{w_{j} !}, \quad B_{q}=\sum_{\substack{v_{\alpha} \geq 0: \\ v_{\alpha}=q, \alpha \in Q \\ w_{j} \geq r_{j}, j \in P}} \prod_{j}\left(w_{j} ! \prod_{\alpha \in R_{j}} \frac{\left|\rho_{\alpha}\right|^{v_{\alpha}}}{v_{\alpha} !}\right)
$$

The $A_{q}$ term is bounded since

$$
A_{q} \leq \prod_{j} \sum_{w_{j}=r_{j}}^{\infty} \frac{G_{j, w_{j}}^{2}}{w_{j} !} \leq \prod_{j} E\left(f_{j}^{2}\right) \leq \sigma^{2} .
$$

If $2 q<r$, there always exists a $j$ in (2.3) such that $w_{j}<r_{j}$, implying (2.4).

For $2 q \geq r$, the multinomial theorem yields

$$
\begin{aligned}
B_{q} & \leq \sum_{\substack{w_{j} \geq r_{j}: \\
\Sigma w_{j}=2 q, j \in P}} \sum_{\substack{v_{\alpha} \geq 0: \Sigma v_{\alpha}=w_{j} \\
\alpha \in R_{j}, j \in P}} \prod_{j}\left(w_{j} ! \prod_{\alpha \in R_{j}} \frac{\left|\rho_{\alpha}\right|^{v_{\alpha}}}{v_{\alpha} !}\right) \\
& \leq \sum_{\substack{w_{j} \geq r_{j}: \\
\Sigma w_{j}=2 q, j \in P}} \prod_{j} \sum_{\substack{v_{\alpha} \geq 0: \\
v_{\alpha}=w_{j}, \alpha \in R_{j}}} w_{j} ! \prod_{\alpha \in R_{j}} \frac{\left|\rho_{\alpha}\right|^{v_{\alpha}}}{v_{\alpha} !} \leq \sum_{\substack{w_{j} \geq r_{j}: \\
\Sigma w_{j}=2 q, j \in P}} \prod_{j}\left(\sum_{\alpha \in R_{j}}\left|\rho_{\alpha}\right|\right)^{w_{j}} \\
& \leq \prod_{j}\left(\sum_{\alpha \in R_{j}}\left|\rho_{\alpha}\right|\right)^{r_{j}} \sum_{\substack{w_{j} \geq 0: \\
\Sigma w_{j}=2 q-r, j \in P}} \prod_{j}\left(\sum_{\alpha \in R_{j}}\left|\rho_{\alpha}\right|\right)^{\sum^{2}}
\end{aligned}
$$




$$
\begin{aligned}
& \leq \prod_{j}\left(\sum_{\alpha \in R_{j}}\left|\rho_{\alpha}\right|\right)^{r_{j}} \sum_{\substack{w_{j} \geq 0: \\
\Sigma w_{j}=2 q-r, j \in P}}(2 q-r) ! \prod_{j} \frac{\left(\sum_{\alpha \in R_{j}}\left|\rho_{\alpha}\right|\right)^{w_{j}}}{w_{j} !} \\
& \leq \prod_{j}\left(\sum_{\alpha \in R_{j}}\left|\rho_{\alpha}\right|\right)^{r_{j}}\left(\sum_{j, k: j \neq k}\left|\rho_{j k}\right|\right)^{2 q-r} \leq \prod_{j}\left(\sum_{\alpha \in R_{j}}\left|\rho_{\alpha}\right|\right)^{r_{j}} \tau^{2 q-r} .
\end{aligned}
$$

Using (A.4), (A.5) in (A.3) gives (2.5). Then (2.6) follows from $\tau<1$.

\section{Appendix B: Propositions for Theorem 2}

We denote the Dirichlet kernel by $D_{m}(\lambda)=\sum_{j=1}^{m} e^{i j \lambda}$, for $m \geq 1$, and will use the fact that

$$
D_{n}\left(\lambda_{j}\right)=n 1(j=0, \bmod n) .
$$

We also use the abbreviating notation

$$
S_{m}(a, b)=E\left\{\hat{F}_{a b}\left(\lambda_{m}\right)\right\}=\frac{1}{n^{2}} \sum_{s, t=1}^{n} \operatorname{Cov}\left(a_{s}, b_{t}\right) D_{m}\left(\lambda_{t-s}\right),
$$

from (B.1), and

$$
S_{m}^{\prime}\left(a, b ; a^{\prime}, b^{\prime}\right)=\frac{1}{n^{2}} \sum_{s, t=1}^{n} \operatorname{Cov}\left(a_{s}, b_{t}\right) \operatorname{Cov}\left(a_{s}^{\prime}, b_{t}^{\prime}\right) D_{m}\left(\lambda_{t-s}\right),
$$

where $a_{t}, b_{t}, a_{t}^{\prime}, b_{t}^{\prime}, t=1, \ldots, n$ are scalar sequences with finite second moments.

Proposition 1 Under (1.2) and Assumptions 1 and 2,

$$
\left(\frac{m}{n}\right)^{2 d-1} E\left\{\hat{F}_{X X}\left(\lambda_{m}\right)\right\} \rightarrow C^{*} \text { as } n \rightarrow \infty
$$


where

$$
\begin{aligned}
& C^{*}=2 \frac{(2 \pi)^{-2 d} \Gamma(2 d)}{1-2 d} \sin \left\{(1-2 d) \frac{\pi}{2}\right\} \beta_{2}^{2 p} E\left(\eta_{1}^{p}\right)^{2} E\left\{g^{p}\left(\eta_{2 t}\right) \eta_{2 t}\right\}^{2} C_{\rho}>0, \\
& C_{\rho}=\lim _{j \rightarrow \infty} E\left(\eta_{20} \eta_{2 j}\right) j^{1-2 d} .
\end{aligned}
$$

Proof. Write

$$
X_{t}=\sum_{j=0}^{p} A_{j t} B_{j t}, \quad A_{j t}=\left(\begin{array}{c}
p \\
j
\end{array}\right) \beta_{2}^{j} \eta_{1 t}^{j} \nu_{1 t}^{p-j}, \quad B_{j t}=g_{t}^{j} h_{t}^{p-j}
$$

Using Lemma 2, since $\left\{A_{j t}\right\}$ is independent of $\left\{B_{k t}\right\}$, for any $j$ and $k$,

$$
\begin{aligned}
\operatorname{Cov}\left(X_{s}, X_{t}\right) & =\sum \operatorname{Cov}\left(A_{j s} B_{j s}, A_{k t} B_{k t}\right) \\
& =\sum\left\{E\left(A_{j s}\right) E\left(A_{k t}\right) \operatorname{Cov}\left(B_{j s}, B_{k t}\right)+\operatorname{Cov}\left(A_{j s}, A_{k t}\right) E\left(B_{j s} B_{k t}\right)\right\},
\end{aligned}
$$

where $\sum$ denotes $\sum_{j, k=0}^{p}$ throughout the proof.

Now define $a_{j}=E\left(A_{j t}\right), b_{g, j}=a_{j} E\left(h_{t}^{p-j}\right)$, and $b_{h, j}=a_{j} E\left(g_{t}^{j}\right)$. Since $\left\{A_{j t}\right\}$ is iid, using Lemma 2 again, for $s \neq t, \operatorname{Cov}\left(X_{s}, X_{t}\right)$ is

$$
\begin{aligned}
\sum a_{j} a_{k} \operatorname{Cov}\left(B_{j s}, B_{k t}\right)=\sum & \left\{b_{g, j} b_{g, k} \operatorname{Cov}\left(g_{s}^{j}, g_{t}^{k}\right)+b_{h, j} b_{h, k} \operatorname{Cov}\left(h_{s}^{p-j}, h_{t}^{p-k}\right)\right. \\
& \left.+a_{j} a_{k} \operatorname{Cov}\left(g_{s}^{j}, g_{t}^{k}\right) \operatorname{Cov}\left(h_{s}^{p-j}, h_{t}^{p-k}\right)\right\} .
\end{aligned}
$$

For $s=t$, denote by $\Lambda$ the difference between $\operatorname{Var}\left(X_{t}\right)$ and (B.2). It follows that $E\left\{\hat{F}_{X X}\left(\lambda_{m}\right)\right\}$ is

$$
\sum\left\{b_{g, j} b_{g, k} S_{m}\left(g^{j}, g^{k}\right)+b_{h, j} b_{h, k} S_{m}\left(h^{p-j}, h^{p-k}\right)+a_{j} a_{k} S_{m}^{\prime}\left(g^{j}, g^{k} ; h^{p-j}, h^{p-k}\right)\right\}+\frac{m}{n} \Lambda .
$$


From (3.7), (3.8), and Lemma $4, b_{g, j} b_{g, k} S_{m}\left(g^{j}, g^{k}\right)=o\left((m / n)^{1-2 d}\right)$, if either $j<p$ or $k<$ $p$, while $b_{g, p}^{2} S_{m}\left(g^{p}, g^{p}\right)=C^{*}(m / n)^{1-2 d}+o\left((m / n)^{1-2 d}\right)$. Lemma 4 and $d^{\prime}<d$ imply that $b_{h, j} b_{h, k} S_{m}\left(h^{p-j}, h^{p-k}\right)=o\left((m / n)^{1-2 d}\right)$, and by Lemma $5, a_{j} a_{k} S_{m}^{\prime}\left(g^{j}, g^{k} ; h^{p-j}, h^{p-k}\right)=$ $o\left((m / n)^{1-2 d}\right)$.

Proposition 2 Under (1.1), (1.2), and Assumptions 1 and 2,

$$
E\left\{\hat{F}_{U U}\left(\lambda_{m}\right)\right\}=O\left(\left(\frac{m}{n}\right)^{1-2 d_{u}}\right)
$$

Proof. Write $U_{t}=\sum_{j=0}^{p-1} A_{\varepsilon, j t} B_{\varepsilon, j t}-A_{\delta, j t} B_{\delta, j t}$, where

$$
A_{\varepsilon, j t}=\left(\begin{array}{c}
p \\
j
\end{array}\right) \beta_{2}^{j} \beta^{j} \eta_{1 t}^{j} \xi_{1 t}^{p-j}, \quad A_{\delta, j t}=\left(\begin{array}{c}
p \\
j
\end{array}\right) \beta_{2}^{j} \beta^{p} \eta_{1 t}^{j} \nu_{1 t}^{p-j}, \quad B_{\varepsilon, j t}=g_{t}^{j} l_{t}^{p-j}, \quad B_{\delta, j t}=g_{t}^{j} h_{t}^{p-j}
$$

Using Lemma 2 repeatedly, since $\left\{A_{\varepsilon, j t}\right\},\left\{A_{\delta, j t}\right\}$ are independent of $\left\{B_{\varepsilon, k t}\right\},\left\{B_{\delta, k t}\right\}$, for any $j$ and $k$,

$$
\begin{aligned}
\operatorname{Cov}\left(U_{s}, U_{t}\right)=\sum & \left\{\operatorname{Cov}\left(A_{\varepsilon, j s} B_{\varepsilon, j s}, A_{\varepsilon, k t} B_{\varepsilon, k t}\right)+\operatorname{Cov}\left(A_{\delta, j s} B_{\delta, j s}, A_{\delta, k t} B_{\delta, k t}\right)\right. \\
& \left.-\operatorname{Cov}\left(A_{\varepsilon, j s} B_{\varepsilon, j s}, A_{\delta, k t} B_{\delta, k t}\right)-\operatorname{Cov}\left(A_{\delta, j s} B_{\delta, j s}, A_{\varepsilon, k t} B_{\varepsilon, k t}\right)\right\} \\
=\sum\{ & E\left(A_{\varepsilon, j s}\right) E\left(A_{\varepsilon, k t}\right) \operatorname{Cov}\left(B_{\varepsilon, j s}, B_{\varepsilon, k t}\right)+\operatorname{Cov}\left(A_{\varepsilon, j s}, A_{\varepsilon, k t}\right) E\left(B_{\varepsilon, j s} B_{\varepsilon, k t}\right) \\
+ & E\left(A_{\delta, j s}\right) E\left(A_{\delta, k t}\right) \operatorname{Cov}\left(B_{\delta, j s}, B_{\delta, k t}\right)+\operatorname{Cov}\left(A_{\delta, j s}, A_{\delta, k t}\right) E\left(B_{\delta, j s} B_{\delta, k t}\right) \\
& -E\left(A_{\varepsilon, j s}\right) E\left(A_{\delta, k t}\right) \operatorname{Cov}\left(B_{\varepsilon, j s}, B_{\delta, k t}\right)-\operatorname{Cov}\left(A_{\varepsilon, j s}, A_{\delta, k t}\right) E\left(B_{\varepsilon, j s} B_{\delta, k t}\right) \\
& \left.-E\left(A_{\delta, j s}\right) E\left(A_{\varepsilon, k t}\right) \operatorname{Cov}\left(B_{\delta, j s}, B_{\varepsilon, k t}\right)-\operatorname{Cov}\left(A_{\delta, j s}, A_{\varepsilon, k t}\right) E\left(B_{\delta, j s} B_{\varepsilon, k t}\right)\right\},
\end{aligned}
$$

where $\sum$ denotes $\sum_{j, k=0}^{p-1}$ throughout the proof.

Now define $a_{\varepsilon j}=E\left(A_{\varepsilon, j t}\right), a_{\delta j}=E\left(A_{\delta, j t}\right), b_{g j}=a_{\varepsilon j} E\left(l_{t}^{p-j}\right)-a_{\delta j} E\left(h_{t}^{p-j}\right), b_{h j}=$ $a_{\delta j} E\left(g_{t}^{j}\right)$, and $b_{l j}=a_{\varepsilon j} E\left(g_{t}^{j}\right)$. Since $\left\{A_{\varepsilon, j t}\right\},\left\{A_{\delta, j t}\right\}$ are jointly iid, using Lemma 2 again, 
for $s \neq t, \operatorname{Cov}\left(U_{s}, U_{t}\right)$ is

$$
\begin{aligned}
\sum & \left\{a_{\varepsilon j} a_{\varepsilon k} \operatorname{Cov}\left(B_{\varepsilon, j s}, B_{\varepsilon, k t}\right)+a_{\delta j} a_{\delta k} \operatorname{Cov}\left(B_{\delta, j s}, B_{\delta, k t}\right)\right. \\
& \left.-a_{\varepsilon j} a_{\delta k} \operatorname{Cov}\left(B_{\varepsilon, j s}, B_{\delta, k t}\right)-a_{\delta j} a_{\varepsilon k} \operatorname{Cov}\left(B_{\delta, j s}, B_{\varepsilon, k t}\right)\right\} \\
=\sum & \left\{b_{g j} b_{g k} \operatorname{Cov}\left(g_{s}^{j}, g_{t}^{k}\right)+b_{h j} b_{h k} \operatorname{Cov}\left(h_{s}^{p-j}, h_{t}^{p-k}\right)+b_{l j} b_{l k} \operatorname{Cov}\left(l_{s}^{p-j}, l_{t}^{p-k}\right)\right. \\
& \left.+a_{\delta j} a_{\delta k} \operatorname{Cov}\left(g_{s}^{j}, g_{t}^{k}\right) \operatorname{Cov}\left(h_{s}^{p-j}, h_{t}^{p-k}\right)+a_{\varepsilon j} a_{\varepsilon k} \operatorname{Cov}\left(g_{s}^{j}, g_{t}^{k}\right) \operatorname{Cov}\left(l_{s}^{p-j}, l_{t}^{p-k}\right)\right\} .
\end{aligned}
$$

For $s=t$, denote by $\Lambda$ the difference between $\operatorname{Var}\left(U_{t}\right)$ and (B.3). It follows that $E\left\{\hat{F}_{U U}\left(\lambda_{m}\right)\right\}$ is

$$
\begin{aligned}
\sum & \left\{b_{g j} b_{g k} S_{m}\left(g^{j}, g^{k}\right)+b_{h j} b_{h k} S_{m}\left(h^{p-j}, h^{p-k}\right)+b_{l j} b_{l k} S_{m}\left(l^{p-j}, l^{p-k}\right)\right. \\
& \left.+a_{\delta j} a_{\delta k} S_{m}^{\prime}\left(g^{j}, g^{k} ; h^{p-j}, h^{p-k}\right)+a_{\varepsilon j} a_{\varepsilon k} S_{m}^{\prime}\left(g^{j}, g^{k} ; l^{p-j}, l^{p-k}\right)\right\}+\frac{m}{n} \Lambda .
\end{aligned}
$$

By (4.5), applying Lemma 4 to each $(j, k)$ pair with non-zero coefficient,

$$
b_{g j} b_{g k} S_{m}\left(g^{j}, g^{k}\right)=O\left((m / n)^{1-2 \max \left\{d_{g}^{*}, 0\right\}}(\log n)^{1\left(d_{g}^{*}=0\right)}\right) .
$$

Similarly, by (4.6) and (4.7), Lemma 4 yields

$$
\begin{aligned}
b_{h j} b_{h k} S_{m}\left(h^{p-j}, h^{p-k}\right) & =O\left((m / n)^{1-2 \max \left\{d_{h}^{*}, 0\right\}}(\log n)^{1\left(d_{h}^{*}=0\right)}\right), \\
b_{l j} b_{l k} S_{m}\left(l^{p-j}, l^{p-k}\right) & =O\left((m / n)^{1-2 \max \left\{d_{l}^{*}, 0\right\}}(\log n)^{1\left(d_{l}^{*}=0\right)}\right) .
\end{aligned}
$$

Finally, Lemma 5, (4.8), and (4.9) give

$$
\begin{aligned}
a_{\delta j} a_{\delta k} S_{m}^{\prime}\left(g^{j}, g^{k} ; h^{p-j}, h^{p-k}\right) & =O\left((m / n)^{1-2 \max \left\{d_{g h}^{*}, 0\right\}}(\log n)^{1\left(d_{g h}^{*}=0\right)}\right) \\
a_{\varepsilon j} a_{\varepsilon k} S_{m}^{\prime}\left(g^{j}, g^{k} ; l^{p-j}, l^{p-k}\right) & =O\left((m / n)^{1-2 \max \left\{d_{g l}^{*}, 0\right\}}(\log n)^{1\left(d_{g l}^{*}=0\right)}\right) .
\end{aligned}
$$


By $(3.8), d_{g}^{*}<d$. Since $d_{h}^{*}$ and $d_{g h}^{*}$ are bounded by $d^{\prime}<d$ while $d_{l}^{*}$ and $d_{g l}^{*}$ are bounded by $d^{\prime \prime}<d$, we have $d^{*}<d$. The bound for $d^{*}=0$ follows from Assumption 2 .

Proposition 3 Under (1.2) and Assumptions 1 and 2,

$$
\operatorname{Var}\left\{\hat{F}_{X X}\left(\lambda_{m}\right)\right\}=o\left(\left(\frac{m}{n}\right)^{2-4 d}\right)
$$

Proof. Define $\rho_{t}=E\left(\eta_{20} \eta_{2 t}\right)$; wherever time indexes $t_{i}, i=1, \ldots, 4$ are used, it will be convenient to write also $\gamma_{i j}=\rho_{t_{j}-t_{i}}$. Denoting $Z_{t}=X_{t}-E\left(X_{t}\right)$, there exists a Gaussian $I(d)$ process $V_{t}$ such that the bounds in Lemma 6 hold. Lemmas 7 and 10 in Robinson (1994b) and Lemma 7 imply that $\operatorname{Var}\left\{\hat{F}_{V V}\left(\lambda_{m}\right)\right\}=o\left((m / n)^{2-4 d}\right)$, so we need to show that the approximation error satisfies

$$
A=\operatorname{Var}\left\{\hat{F}_{X X}\left(\lambda_{m}\right)\right\}-\operatorname{Var}\left\{\hat{F}_{V V}\left(\lambda_{m}\right)\right\}=o\left(\left(\frac{m}{n}\right)^{2-4 d}\right)
$$

Since $n^{2}\left[\hat{F}_{X X}\left(\lambda_{m}\right)-E\left\{\hat{F}_{X X}\left(\lambda_{m}\right)\right\}\right]$ can be written

$$
\sum_{t_{1}, t_{2}=1}^{n}\left\{X_{t_{1}} X_{t_{2}}-E\left(X_{t_{1}} X_{t_{2}}\right)\right\} D_{m}\left(\lambda_{t_{2}-t_{1}}\right)=\sum_{t_{1}, t_{2}=1}^{n}\left\{Z_{t_{1}} Z_{t_{2}}-E\left(Z_{t_{1}} Z_{t_{2}}\right)\right\} D_{m}\left(\lambda_{t_{2}-t_{1}}\right)
$$

by (B.1), we have

$$
\operatorname{Var}\left\{\hat{F}_{X X}\left(\lambda_{m}\right)\right\}=\frac{1}{n^{4}} \sum_{t_{1}, t_{2}, t_{3}, t_{4}=1}^{n} \operatorname{Cov}\left(Z_{t_{1}} Z_{t_{2}}, Z_{t_{3}} Z_{t_{4}}\right) D_{m}\left(\lambda_{t_{2}-t_{1}}\right) \overline{D_{m}\left(\lambda_{t_{4}-t_{3}}\right)}
$$

and therefore

$$
A=\frac{1}{n^{4}} \sum_{t_{1}, t_{2}, t_{3}, t_{4}=1}^{n}\left\{\operatorname{Cov}\left(Z_{t_{1}} Z_{t_{2}}, Z_{t_{3}} Z_{t_{4}}\right)-\operatorname{Cov}\left(V_{t_{1}} V_{t_{2}}, V_{t_{3}} V_{t_{4}}\right)\right\} D_{m}\left(\lambda_{t_{2}-t_{1}}\right) \overline{D_{m}\left(\lambda_{t_{4}-t_{3}}\right)}
$$


We now decompose $A$ into sums where the time indexes conform to cases (a) to (g) in Lemma 6. Using Lemmas 3 and 6 repeatedly, the approximation error for each case is bounded by:

(a)

$$
\sum_{\alpha_{1}, \alpha_{2} \in Q_{4}: \alpha_{1} \neq \alpha_{2}} \frac{K}{n^{4}} \sum_{t_{1}, t_{2}, t_{3}, t_{4}=1}^{n} \gamma_{\alpha_{1}}^{2}\left|\gamma_{\alpha_{2}}\right|\left|D_{m}\left(\lambda_{t_{2}-t_{1}}\right)\right|\left|D_{m}\left(\lambda_{t_{4}-t_{3}}\right)\right| .
$$

If $\alpha_{1}$ is either $(1,2)$ or $(3,4)$, each element in the first summation is bounded by

$$
\begin{aligned}
& \frac{K}{n^{2}} \sum_{j=1}^{n} \rho_{j}^{2}\left|D_{m}\left(\lambda_{j}\right)\right| \sum_{j=1}^{n}\left|\rho_{j}\right|\left|D_{m}\left(\lambda_{j}\right)\right|+\frac{K}{n^{3}} \sum_{j=1}^{n} \rho_{j}^{2}\left|D_{m}\left(\lambda_{j}\right)\right| \sum_{j=1}^{n}\left|\rho_{j}\right| \sum_{j=1}^{n}\left|D_{m}\left(\lambda_{j}\right)\right| \\
\leq & K\left(\frac{m}{n}\right)^{2-2 d}\left(1+\frac{\log m}{m^{1-2 d}}\right)\left\{1+(\log n) 1\left(d=\frac{1}{4}\right)+\left(\frac{n}{m}\right)^{4 d-1} 1\left(d>\frac{1}{4}\right)\right\},
\end{aligned}
$$

while if $\alpha_{1}$ is not equal to $(1,2)$ or to $(3,4)$, we have a bound

$$
\begin{aligned}
& \frac{K}{n^{3}} \sum_{j=1}^{n} \rho_{j}^{2} \sum_{j=1}^{n}\left|\rho_{j}\right|\left|D_{m}\left(\lambda_{j}\right)\right| \sum_{j=1}^{n}\left|D_{m}\left(\lambda_{j}\right)\right|+\frac{K}{n^{4}} \sum_{j=1}^{n} \rho_{j}^{2} \sum_{j=1}^{n}\left|\rho_{j}\right|\left\{\sum_{j=1}^{n}\left|D_{m}\left(\lambda_{j}\right)\right|\right\}^{2} \\
\leq & K\left(\frac{m}{n}\right)^{2-2 d} \frac{\log m}{m}\left(1+\frac{\log m}{m^{1-2 d}}\right)\left\{1+(\log n) 1\left(d=\frac{1}{4}\right)+n^{4 d-1} 1\left(d>\frac{1}{4}\right)\right\} .
\end{aligned}
$$

$$
\begin{aligned}
& \frac{K}{n^{4}} \sum_{t_{1}, t_{2}, t_{3}=1}^{n}\left(\gamma_{12}^{2}+\gamma_{13}^{2}+\gamma_{23}^{2}\right)\left|D_{m}\left(\lambda_{t_{2}-t_{1}}\right)\right|\left|D_{m}\left(\lambda_{t_{3}-t_{1}}\right)\right| \\
\leq & \frac{K}{n^{3}} \sum_{j=1}^{n} \rho_{j}^{2}\left|D_{m}\left(\lambda_{j}\right)\right| \sum_{j=1}^{n}\left|D_{m}\left(\lambda_{j}\right)\right|+\frac{K}{n^{4}} \sum_{j=1}^{n} \rho_{j}^{2}\left\{\sum_{j=1}^{n}\left|D_{m}\left(\lambda_{j}\right)\right|\right\}^{2} \\
\leq & K\left(\frac{m}{n}\right)^{2} \frac{\log m}{m}\left[\left(1+\frac{\log m}{m}\right)\left\{1+(\log n) 1\left(d=\frac{1}{4}\right)\right\}\right. \\
& \left.+\left\{\left(\frac{n}{m}\right)^{4 d-1}+n^{4 d-1} \frac{\log m}{m}\right\} 1\left(d>\frac{1}{4}\right)\right] .
\end{aligned}
$$


(c)

$$
\begin{aligned}
& \frac{K}{n^{4}} \sum_{t_{1}, t_{2}, t_{3}=1}^{n}\left(\gamma_{12}^{2}+\gamma_{13}^{2}+\gamma_{23}^{2}\right)\left|D_{m}(0)\right|\left|D_{m}\left(\lambda_{t_{3}-t_{2}}\right)\right| \\
\leq & K \frac{m}{n^{2}} \sum_{j=1}^{n} \rho_{j}^{2}\left|D_{m}\left(\lambda_{j}\right)\right|+K \frac{m}{n^{3}} \sum_{j=1}^{n} \rho_{j}^{2} \sum_{j=1}^{n}\left|D_{m}\left(\lambda_{j}\right)\right| \\
\leq & K\left(\frac{m}{n}\right)^{2}\left[\left(1+\frac{\log m}{n}\right)\left\{1+(\log n) 1\left(d=\frac{1}{4}\right)\right\}\right. \\
& \left.+\left\{\left(\frac{n}{m}\right)^{4 d-1}+n^{4 d-1} \frac{\log m}{n}\right\} 1\left(d>\frac{1}{4}\right)\right] .
\end{aligned}
$$

(d), (e), (f) For any $a=a\left(t_{1}, t_{2}\right)$ and $b=b\left(t_{1}, t_{2}\right)$,

$$
\frac{K}{n^{4}} \sum_{t_{1}, t_{2}=1}^{n}\left|\gamma_{12}\right|\left|D_{m}(a)\right|\left|D_{m}(b)\right| \leq K \frac{m^{2}}{n^{3}} \sum_{j=1}^{n}\left|\rho_{j}\right| \leq K\left(\frac{m}{n}\right)^{2} n^{2 d-1}
$$

$(\mathrm{g})$

$$
\frac{K}{n^{4}} \sum_{t_{1}=1}^{n}\left|D_{m}(0)\right|^{2} \leq K\left(\frac{m}{n}\right)^{2} n^{-1}
$$

Since cases (a) to (g) satisfy (B.4), the proof is complete.

\section{Appendix C: Technical lemmas for Appendix B}

Lemma 1 Let $\left|\rho_{j}-\rho_{j+1}\right| \leq K\left|\rho_{j+1}\right| / j$ and $\left|\gamma_{j}-\gamma_{j+1}\right| \leq K\left|\gamma_{j+1}\right| / j$, for all $j \geq 1$. Then, for any positive integers $r, s$, and $j$,

$$
\begin{gathered}
\left|\rho_{j}^{r}-\rho_{j+1}^{r}\right| \leq K \frac{\left|\rho_{j+1}^{r}\right|}{j} \\
\left|\rho_{j}^{r} \gamma_{j}^{s}-\rho_{j+1}^{r} \gamma_{j+1}^{s}\right| \leq K \frac{\left|\rho_{j+1}^{r} \gamma_{j+1}^{s}\right|}{j} .
\end{gathered}
$$


Proof. First note that

$$
(a-b)^{k}=\sum_{i=0}^{k}\left(\begin{array}{l}
k \\
i
\end{array}\right) a^{i}(-b)^{k-i}=\sum_{i=0}^{k}\left(\begin{array}{l}
k \\
i
\end{array}\right)\left(a^{i}-b^{i}\right)(-b)^{k-i},
$$

since

$$
\sum_{i=0}^{k}\left(\begin{array}{l}
k \\
i
\end{array}\right) b^{i}(-b)^{k-i}=(b-b)^{k}=0
$$

Hence,

$$
\begin{aligned}
\left|a^{k}-b^{k}\right| & =\left|(a-b)^{k}-\sum_{i=1}^{k-1}\left(\begin{array}{c}
k \\
i
\end{array}\right)\left(a^{i}-b^{i}\right)(-b)^{k-i}\right| \\
& \leq|a-b|^{k}+\sum_{i=1}^{k-1}\left(\begin{array}{c}
k \\
i
\end{array}\right)|b|^{k-i}\left|a^{i}-b^{i}\right| .
\end{aligned}
$$

Proceeding by induction, suppose (C.1) holds for $r=1,2, \ldots, k-1$. Then

$$
\begin{aligned}
\left|\rho_{j}^{k}-\rho_{j+1}^{k}\right| & \leq\left|\rho_{j}-\rho_{j+1}\right|^{k}+\sum_{i=1}^{k-1}\left(\begin{array}{c}
k \\
i
\end{array}\right)\left|\rho_{j+1}^{k-i}\right|\left|\rho_{j}^{i}-\rho_{j+1}^{i}\right| \\
& \leq K \frac{\left|\rho_{j+1}^{k}\right|}{j^{k}}+K \sum_{i=1}^{k-1}\left|\rho_{j+1}^{k-i}\right| \frac{\left|\rho_{j+1}^{i}\right|}{j} \leq K \frac{\left|\rho_{j+1}^{k}\right|}{j}
\end{aligned}
$$

proving (C.1). To prove (C.2) we use (C.1):

$$
\begin{aligned}
\left|\rho_{j}^{r} \gamma_{j}^{s}-\rho_{j+1}^{r} \gamma_{j+1}^{s}\right| & =\left|\left(\rho_{j}^{r}-\rho_{j+1}^{r}\right)\left(\gamma_{j}^{s}-\gamma_{j+1}^{s}\right)+\gamma_{j+1}^{s}\left(\rho_{j}^{r}-\rho_{j+1}^{r}\right)+\rho_{j+1}^{r}\left(\gamma_{j}^{s}-\gamma_{j+1}^{s}\right)\right| \\
& \leq K \frac{\left|\rho_{j+1}^{r}\right|}{j} \frac{\left|\gamma_{j+1}^{s}\right|}{j}+K\left|\gamma_{j+1}^{s}\right| \frac{\left|\rho_{j+1}^{r}\right|}{j}+K\left|\rho_{j+1}^{r}\right| \frac{\left|\gamma_{j+1}^{s}\right|}{j} \leq K \frac{\left|\rho_{j+1}^{r} \gamma_{j+1}^{s}\right|}{j}
\end{aligned}
$$

Lemma 2 If $\left(a_{1}, b_{1}\right)$ is independent of $\left(a_{2}, b_{2}\right)$ and $E\left(a_{i}^{2}+b_{i}^{2}\right)<\infty$, 


$$
\begin{aligned}
& \operatorname{Cov}\left(a_{1} a_{2}, b_{1} b_{2}\right)=\operatorname{Cov}\left(a_{1}, b_{1}\right) E\left(a_{2}\right) E\left(b_{2}\right)+E\left(a_{1} b_{1}\right) \operatorname{Cov}\left(a_{2}, b_{2}\right) \\
& =\operatorname{Cov}\left(a_{1}, b_{1}\right) E\left(a_{2}\right) E\left(b_{2}\right)+E\left(a_{1}\right) E\left(b_{1}\right) \operatorname{Cov}\left(a_{2}, b_{2}\right)+\operatorname{Cov}\left(a_{1}, b_{1}\right) \operatorname{Cov}\left(a_{2}, b_{2}\right) .
\end{aligned}
$$

Proof. Straightforward.

Lemma 3 Let $\rho_{j}=O\left(j^{2 d-1}\right), a>0, b \geq 1, m \leq n / 2$, and $d^{+}=(a-1) / 2 a$. Then,

$$
\begin{aligned}
\sum_{j=1}^{n}\left|\rho_{j}\right|^{a} & =O\left(1+(\log n) 1\left(d=d^{+}\right)+n^{a(2 d-1)+1} 1\left(d>d^{+}\right)\right), \\
\sum_{j=1}^{n}\left|D_{m}\left(\lambda_{j}\right)\right|^{b} & =O\left(n\left\{\log m+m^{b-1} 1(b>1)\right\}\right), \\
\sum_{j=1}^{n}\left|\rho_{j}\right|^{a}\left|D_{m}\left(\lambda_{j}\right)\right|^{b} & =O\left(m^{b}\left\{1+(\log n) 1\left(d=d^{+}\right)+\left(\frac{n}{m}\right)^{a(2 d-1)+1} 1\left(d>d^{+}\right)\right\}\right) .
\end{aligned}
$$

Proof. From e.g. Zygmund (1977, p. 11) and an elementary inequality,

$$
\left|D_{m}\left(\lambda_{j}\right)\right| \leq K \min \left\{m, \frac{n}{|j|}\right\}, \quad|j| \leq \frac{n}{2}
$$

The remainder of the proof is straightforward.

Lemma 4 For $j=1,2$, define $g_{j, t}=g_{j}\left(\mu_{t}\right)$, where $\mu_{t}$ is a standard Gaussian $I(d)$ process and $\rho_{t}=E\left(\mu_{0} \mu_{t}\right)$. Assume $E\left(g_{j, t}^{2}\right)<\infty$. Denote by $G_{j, k}$ the $k$-th Hermite coefficient of $g_{j}(\cdot)$, and let

$$
r=\min \left\{k \in \mathbb{N}: G_{1, k} G_{2, k} \neq 0\right\} .
$$

If $d>0$, define

$$
d^{*}=\frac{1}{2}-r\left(\frac{1}{2}-d\right), \quad C_{\rho}=\lim _{j \rightarrow \infty} \rho_{j} j^{1-2 d} .
$$


Let $A=S_{m}\left(g_{1}, g_{2}\right)$, where $m$ satisfies Assumption 2 if $d^{*}=1 /(2 r+2)$ or just $(4.4)$ otherwise. Then,

$$
\begin{aligned}
A= & O\left(\frac{m}{n}\left\{1(d=0)+1\left(d^{*}<0\right)+(\log n) 1\left(d^{*}=0\right)\right\}\right) \\
& +\left\{C^{*}\left(\frac{m}{n}\right)^{1-2 d^{*}}+o\left(\left(\frac{m}{n}\right)^{1-2 d^{*}}\right)\right\} 1\left(d^{*}>0\right),
\end{aligned}
$$

where

$$
C^{*}=2 \frac{(2 \pi)^{-2 d^{*}} \Gamma\left(2 d^{*}\right)}{1-2 d^{*}} \frac{G_{1, r} G_{2, r}}{r !} \sin \left\{\left(1-2 d^{*}\right) \frac{\pi}{2}\right\} C_{\rho}^{r} \neq 0
$$

Proof. Let $\gamma_{t}=\operatorname{Cov}\left(g_{1,0} ; g_{2, t}\right)$. Then

$$
A=\frac{1}{n^{2}} \sum_{s, t=1}^{n} \gamma_{t-s} D_{m}\left(\lambda_{t-s}\right)=\frac{1}{n} \sum_{u=1-n}^{n-1}\left(1-\frac{|u|}{n}\right) \gamma_{u} D_{m}\left(\lambda_{u}\right)
$$

We will make repeated use of (C.3) and of $\rho_{u}^{r}=K u^{r(2 d-1)}=O\left(u^{2 d^{*}-1}\right)$. By Theorem 1 and (C.4),

$$
\gamma_{u}=\sum_{k=1}^{\infty} \frac{G_{1, k} G_{2, k}}{k !} \rho_{u}^{k}=C \rho_{u}^{r}+O\left(\left|\rho_{u}^{r+1}\right|\right)
$$

where $C=G_{1, r} G_{2, r} / r !$.

(a) If $d=0$, then $\gamma_{u}=O\left(\left|\rho_{u}^{r}\right|\right)$ are summable. Similarly, if $d^{*}<0$, then $\gamma_{u}=$ $O\left(\left|\rho_{u}^{r}\right|\right)=O\left(u^{2 d^{*}-1}\right)$ are summable. In either case,

$$
A \leq \frac{K}{n} \sum_{u=1-n}^{n-1}\left(1-\frac{|u|}{n}\right)\left|\gamma_{u}\right|\left|D_{m}\left(\lambda_{u}\right)\right| \leq K \frac{m}{n} \sum_{u=1-n}^{n-1}\left|\gamma_{u}\right|=O\left(\frac{m}{n}\right)
$$

(b) If $d^{*}=0, \gamma_{u}=O\left(\left|\rho_{u}^{r}\right|\right)=O\left(u^{-1}\right)$, hence

$$
A \leq K \frac{m}{n} \sum_{u=1-n}^{n-1}\left|\gamma_{u}\right|=O\left(\frac{m}{n} \log n\right)
$$


(c) If $d^{*}>0,\left|\gamma_{u}-C \rho_{u}^{r}\right| \leq K\left|\rho_{u}^{r+1}\right| \leq K\left|\rho_{u}^{r}\right|^{1+\omega}$, where $\omega=r^{-1}$. Defining

$$
B_{1}=\frac{1}{n} \sum_{u=1-n}^{n-1}\left(1-\frac{|u|}{n}\right) \rho_{u}^{r} D_{m}\left(\lambda_{u}\right)
$$

we get

$$
\begin{aligned}
\left|A-C B_{1}\right| & \leq \frac{1}{n} \sum_{u=1-n}^{n-1}\left(1-\frac{|u|}{n}\right)\left|\gamma_{u}-C \rho_{u}^{r}\right|\left|D_{m}\left(\lambda_{u}\right)\right| \\
& \leq \frac{K}{n} \sum_{u=1-n}^{n-1}\left|\rho_{u}^{r}\right|^{1+\omega}\left|D_{m}\left(\lambda_{u}\right)\right| \leq K \frac{m}{n}+\frac{K}{n} \sum_{u=1}^{n}\left|\rho_{u}^{r}\right|^{1+\omega}\left|D_{m}\left(\lambda_{u}\right)\right| .
\end{aligned}
$$

Therefore, setting $d^{+}=\omega /(2+2 \omega)$ in Lemma 3 ,

$$
\begin{aligned}
\left|A-C B_{1}\right| & =O\left(\frac{m}{n}\left\{1+(\log n) 1\left(d^{*}=d^{+}\right)+\left(\frac{m}{n}\right)^{(1+\omega)\left(1-2 d^{*}\right)-1} 1\left(d^{*}>d^{+}\right)\right\}\right) \\
& =o\left(\left(\frac{m}{n}\right)^{1-2 d^{*}}\right),
\end{aligned}
$$

choosing $0<\epsilon<2 d^{*}$ in Assumption 2 if $d^{*}=d^{+}$. Now, write

$$
\begin{aligned}
B_{1} & =\frac{1}{n} \sum_{|u|<n}\left(1-\frac{|u|}{n}\right) \rho_{u}^{r} D_{m}\left(\lambda_{u}\right)=\frac{1}{n} \sum_{|u|<n} \rho_{u}^{r} D_{m}\left(\lambda_{u}\right)-\frac{1}{n^{2}} \sum_{|u|<n}|u| \rho_{u}^{r} D_{m}\left(\lambda_{u}\right) \\
& =\frac{1}{n} \sum_{u=-\infty}^{\infty} \rho_{u}^{r} D_{m}\left(\lambda_{u}\right)-\frac{1}{n} \sum_{|u| \geq n} \rho_{u}^{r} D_{m}\left(\lambda_{u}\right)-\frac{1}{n^{2}} \sum_{|u|<n}|u| \rho_{u}^{r} D_{m}\left(\lambda_{u}\right)=B_{2}+B_{3}+B_{4},
\end{aligned}
$$

where

$$
\begin{aligned}
& B_{2}=\frac{2 \pi}{n} \sum_{j=1}^{m} f\left(\lambda_{j}\right), \quad f\left(\lambda_{j}\right)=\frac{1}{2 \pi} \sum_{u=-\infty}^{\infty} \rho_{u}^{r} e^{i u \lambda_{j}}, \\
& B_{3}=-\frac{1}{n} \sum_{u=n}^{\infty} \rho_{u}^{r}\left\{D_{m}\left(\lambda_{u}\right)+\overline{D_{m}\left(\lambda_{u}\right)}\right\}
\end{aligned}
$$




$$
B_{4}=-\frac{1}{n^{2}} \sum_{u=1}^{n-1} u \rho_{u}^{r}\left\{D_{m}\left(\lambda_{u}\right)+\overline{D_{m}\left(\lambda_{u}\right)}\right\}
$$

Then

$$
\left|A-C B_{2}\right|=\left|A-C\left(B_{1}-B_{3}-B_{4}\right)\right| \leq\left|A-C B_{1}\right|+C\left|B_{3}\right|+C\left|B_{4}\right| .
$$

Note that, for any $u$,

$$
\left|\sum_{k=1}^{u} D_{m}\left(\lambda_{k}\right)\right|=\left|\sum_{k=1}^{u} \sum_{j=1}^{m} e^{i j \lambda_{k}}\right| \leq \sum_{j=1}^{m}\left|D_{u}\left(\lambda_{j}\right)\right| \leq K \sum_{j=1}^{m} \frac{n}{j} \leq K n \log m .
$$

Using summation by parts, (C.13), and Lemma 1 in (C.11),

$$
\begin{aligned}
\left|B_{4}\right| & \leq \frac{K}{n^{2}} \sum_{u=1}^{n-1}\left\{\left|u \rho_{u}^{r}-(u+1) \rho_{u+1}^{r}\right|\left|\sum_{k=1}^{u} D_{m}\left(\lambda_{k}\right)\right|\right\}+\frac{K}{n^{2}}\left|\sum_{j=1}^{n-1} D_{m}\left(\lambda_{j}\right)\right| n\left|\rho_{n}^{r}\right| \\
& \leq \frac{K}{n^{2}} \sum_{u=1}^{n-1}\left(u\left|\rho_{u}^{r}-\rho_{u+1}^{r}\right|+\left|\rho_{u+1}^{r}\right|\right) n \log m+K n^{2 d^{*}-1} \log m \\
& \leq K \frac{\log m}{n} \sum_{u=1}^{n-1} u^{2 d^{*}-1}+K n^{2 d^{*}-1} \log m \leq K n^{2 d^{*}-1} \log m=o\left(\left(\frac{m}{n}\right)^{1-2 d^{*}}\right) .
\end{aligned}
$$

Using the partial summation formula for infinite sums, (C.13), and Lemma 1 in (C.10),

$$
\begin{aligned}
\left|B_{3}\right| & \leq \frac{K}{n} \sum_{u=n}^{\infty}\left|\rho_{u}^{r}-\rho_{u+1}^{r}\right|\left|\sum_{k=1}^{u} D_{m}\left(\lambda_{k}\right)\right|+\frac{K}{n}\left|\sum_{j=1}^{n-1} D_{m}\left(\lambda_{j}\right)\right|\left|\rho_{n}^{r}\right| \\
& \leq K \log m \sum_{u=n}^{\infty} u^{2 d^{*}-2}+K n^{2 d^{*}-1} \log m \leq K n^{2 d^{*}-1} \log m=o\left(\left(\frac{m}{n}\right)^{1-2 d^{*}}\right) .
\end{aligned}
$$


Lemma 1 implies that $f(\lambda) \sim C_{f} \lambda^{-2 d^{*}}$ as $\lambda \rightarrow 0^{+}$, where

$$
C_{f}=\pi^{-1} \Gamma\left(2 d^{*}\right) \sin \left\{\left(1-2 d^{*}\right) \frac{\pi}{2}\right\} C_{\rho}^{r}
$$

Thus, by Proposition 1 in Robinson (1994a),

$$
B_{2}=\frac{2 \pi}{n} \sum_{j=1}^{m} f\left(\lambda_{j}\right) \sim \int_{0}^{\lambda_{m}} f(t) \mathrm{d} t \sim C_{f} \frac{\lambda_{m}^{1-2 d^{*}}}{1-2 d^{*}} \sim C_{f} \frac{(2 \pi)^{1-2 d^{*}}}{1-2 d^{*}}\left(\frac{m}{n}\right)^{1-2 d^{*}}
$$

which together with (C.12), (C.14), (C.15) gives (C.5).

Lemma 5 For $i, j=1,2$, define $g_{i j, t}=g_{i j}\left(\mu_{i t}\right)$, where $\mu_{i t}$ is a standard Gaussian $I\left(d_{i}\right)$ process and $\rho_{i, t}=E\left(\mu_{i 0} \mu_{i t}\right)$. Assume $E\left(g_{i j, t}^{2}\right)<\infty$. Denote by $G_{i j, k}$ the $k$-th Hermite coefficients of $g_{i j}(\cdot)$, with

$$
r_{i}=\min \left\{k>0: G_{i 1, k} G_{i 2, k} \neq 0\right\}
$$

Let $d_{1} \geq d_{2}$ without loss of generality, and define

$$
d^{*}=\frac{1}{2}-r_{1}\left(\frac{1}{2}-d_{1}\right)-r_{2}\left(\frac{1}{2}-d_{2}\right), \quad C_{i \rho}=\lim _{j \rightarrow \infty} \rho_{i j} j^{1-2 d_{i}}
$$

Let $A=S_{m}^{\prime}\left(g_{11}, g_{12} ; g_{21}, g_{22}\right)$, where $m$ satisfies Assumption 2 if $d^{*}+d_{1}=1 / 2$ or just (4.4) otherwise. Then,

$$
\begin{aligned}
A= & O\left(\frac{m}{n}\left\{1+(\log n) 1\left(d^{*}=0\right)\right\}\right) \\
& +\left\{C^{*}\left(\frac{m}{n}\right)^{1-2 d^{*}}+o\left(\left(\frac{m}{n}\right)^{1-2 d^{*}}\right)\right\} 1\left(d^{*}>0\right),
\end{aligned}
$$


where

$$
C^{*}=2 \frac{(2 \pi)^{-2 d^{*}} \Gamma\left(2 d^{*}\right)}{1-2 d^{*}} \frac{G_{11, r_{1}} G_{12, r_{1}}}{r_{1} !} \frac{G_{21, r_{2}} G_{22, r_{2}}}{r_{2} !} \sin \left\{\left(1-2 d^{*}\right) \frac{\pi}{2}\right\} C_{1 \rho}^{r_{1}} C_{2 \rho}^{r_{2}} \neq 0 .
$$

Proof. Let $\gamma_{i, t}=\operatorname{Cov}\left(g_{i 1,0} ; g_{i 2, t}\right)$. Then, similarly to (C.6),

$$
A=\frac{1}{n} \sum_{u=1-n}^{n-1}\left(1-\frac{|u|}{n}\right) \gamma_{1 u} \gamma_{2 u} D_{m}\left(\lambda_{u}\right) .
$$

By Theorem 1 and (C.16),

$$
\gamma_{i u}=\sum_{k=1}^{\infty} \frac{G_{i 1, k} G_{i 2, k}}{k !} \rho_{i u}^{k}=C_{i} \rho_{i u}^{r_{i}}+O\left(\left|\rho_{i u}^{r_{i}+1}\right|\right),
$$

where $C_{i}=G_{i 1, r_{i}} G_{i 2, r_{i}} / r_{i}$ !.

(a) If $d_{1} d_{2}=0$, then $\gamma_{1 u} \gamma_{2 u}=O\left(\left|\rho_{1 u}^{r_{1}} \rho_{2 u}^{r_{2}}\right|\right)$ are summable. Similarly, if $d_{1} d_{2}>0$ but $d^{*}<0$, then $\gamma_{1 u} \gamma_{2 u}=O\left(\left|\rho_{1 u}^{r_{1}} \rho_{2 u}^{r_{2}}\right|\right)=O\left(u^{r_{1}\left(2 d_{1}-1\right)+r_{2}\left(2 d_{2}-1\right)}\right)=O\left(u^{2 d^{*}-1}\right)$ are summable. In either case, writing $\gamma_{1 u} \gamma_{2 u}$ instead of $\gamma_{u}$ in (C.7) yields $A=O(m / n)$.

(b) If $d^{*}=0, \gamma_{1 u} \gamma_{2 u}=O\left(u^{-1}\right)$, hence (C.8) holds for $\gamma_{1 u} \gamma_{2 u}$.

(c) If $d^{*}>0$,

$$
\begin{aligned}
\left|\gamma_{1 u} \gamma_{2 u}-C_{1} C_{2} \rho_{2 u}^{r_{1}} \rho_{2 u}^{r_{2}}\right| & \leq\left|\gamma_{1 u}\right|\left|\gamma_{2 u}-C_{2} \rho_{2 u}^{r_{2}}\right|+C_{2}\left|\rho_{2 u}^{r_{2}}\right|\left|\gamma_{1 u}-C_{1} \rho_{1 u}^{r_{1}}\right| \\
& \leq K\left|\rho_{1 u}^{r_{1}} \rho_{2 u}^{r_{2}+1}\right|+K\left|\rho_{2 u}^{r_{2}} \rho_{1 u}^{r_{1}+1}\right| \leq K\left|\rho_{1 u}^{r_{1}+1} \rho_{1 u}^{r_{2}}\right| \leq K\left|\rho_{1 u}^{r_{1}} \rho_{2 u}^{r_{2}}\right|^{1+\omega}
\end{aligned}
$$

where $\omega=\left(1-2 d_{1}\right) /\left(1-2 d^{*}\right)$, since $d_{1} \geq d_{2}$. Then (C.17) follows from the proof of case (c) of Lemma 4, writing $\rho_{1 u}^{r_{1}} \rho_{2 u}^{r_{2}}$ instead of $\rho_{u}^{r}$.

Lemma $6 \operatorname{Under}(1.2)$ and Assumption 1, let $Z_{t}=X_{t}-E\left(X_{t}\right)$. For $t_{1}, t_{2}, t_{3}, t_{4}$ distinct, define $\rho_{i j}=E\left(\eta_{2 t_{i}} \eta_{2 t_{j}}\right)$ and $\rho_{i j}^{\prime}=E\left(\nu_{2 t_{i}} \nu_{2 t_{j}}\right)$. Then there exists a mean-zero 
Gaussian $I(d)$ process $V_{t}$ such that:

(a) $\operatorname{Cov}\left(Z_{t_{1}} Z_{t_{2}}, Z_{t_{3}} Z_{t_{4}}\right)-\operatorname{Cov}\left(V_{t_{1}} V_{t_{2}}, V_{t_{3}} V_{t_{4}}\right)=O\left(\sum_{\alpha_{1}, \alpha_{2} \in Q_{4}: \alpha_{1} \neq \alpha_{2}} \rho_{\alpha_{1}}^{2}\left|\rho_{\alpha_{2}}\right|\right)$;

(b) $\operatorname{Cov}\left(Z_{t_{1}} Z_{t_{2}}, Z_{t_{1}} Z_{t_{3}}\right)-\operatorname{Cov}\left(V_{t_{1}} V_{t_{2}}, V_{t_{1}} V_{t_{3}}\right)=O\left(\rho_{12}^{2}+\rho_{13}^{2}+\rho_{23}^{2}\right)$;

(c) $\operatorname{Cov}\left(Z_{t_{1}}^{2}, Z_{t_{2}} Z_{t_{3}}\right)-\operatorname{Cov}\left(V_{t_{1}}^{2}, V_{t_{2}} V_{t_{3}}\right)=O\left(\rho_{12}^{2}+\rho_{13}^{2}+\rho_{23}^{2}\right)$;

(d) $\operatorname{Cov}\left(Z_{t_{1}} Z_{t_{2}}, Z_{t_{1}} Z_{t_{2}}\right)-\operatorname{Cov}\left(V_{t_{1}} V_{t_{2}}, V_{t_{1}} V_{t_{2}}\right)=O\left(\left|\rho_{12}\right|\right)$;

(e) $\operatorname{Cov}\left(Z_{t_{1}}^{2}, Z_{t_{2}}^{2}\right)-\operatorname{Cov}\left(V_{t_{1}}^{2}, V_{t_{2}}^{2}\right)=O\left(\left|\rho_{12}\right|\right)$;

(f) $\operatorname{Cov}\left(Z_{t_{1}}^{2}, Z_{t_{1}} Z_{t_{2}}\right)-\operatorname{Cov}\left(V_{t_{1}}^{2}, V_{t_{1}} V_{t_{2}}\right)=O\left(\left|\rho_{12}\right|\right)$;

(g) $\operatorname{Cov}\left(Z_{t_{1}}^{2}, Z_{t_{1}}^{2}\right)-\operatorname{Cov}\left(V_{t_{1}}^{2}, V_{t_{1}}^{2}\right)=O(1)$.

Proof. All $Z_{t}$ covariances in (a) to (g) can be written as linear combinations of

$$
\begin{array}{r}
E\left(\prod_{i=1}^{4} Z_{t_{i}}^{k_{i}}\right)=E\left\{E\left(\prod_{i=1}^{4} Z_{t_{i}}^{k_{i}} \mid g_{t_{j}}, h_{t_{j}}, j=1, \ldots, 4\right)\right\} \\
=E\left\{\prod_{i=1}^{4} E\left(Z_{t_{i}}^{k_{i}} \mid g_{t_{j}}, h_{t_{j}}, j=1, \ldots, 4\right)\right\}=E\left\{\prod_{i=1}^{4} E\left(Z_{t_{i}}^{k_{i}} \mid g_{t_{i}}, h_{t_{i}}\right)\right\},
\end{array}
$$

where, conditionally on $g_{s}$ and $h_{s}, Z_{s}$ is independent of $Z_{t}, g_{t}$, and $h_{t}$, for any $t \neq s$.

In what follows, let $s_{i}, i=1, \ldots, 4$ denote (not necessarily distinct) elements of $\left\{t_{1}, t_{2}, t_{3}, t_{4}\right\}$. Wherever $u_{i}$ and $s_{i}$ are both defined, let

$$
A_{i}=\left(\begin{array}{c}
p \\
u_{i}
\end{array}\right) \beta_{2}^{u_{i}} \eta_{1 s_{i}}^{u_{i}} \nu_{1 s_{i}}^{p-u_{i}}, \quad B_{i}=g_{s_{i}}^{u_{i}} h_{s_{i}}^{p-u_{i}}
$$

and define $c_{i}=E\left(A_{i}\right), c_{i j}=E\left(\tilde{A}_{i} \tilde{A}_{j}\right), c_{i j k}=E\left(\tilde{A}_{i} \tilde{A}_{j} \tilde{A}_{k}\right)$, where throughout the proof $\tilde{z}_{t}^{u}=z_{t}^{u}-E\left(z_{t}^{u}\right)$. We first compute $E\left(Z_{t}^{k} \mid g_{t}, h_{t}\right)$ for $k=1, \ldots, 3$. Setting $s_{1}=s_{2}=s_{3}=t$, but omitting time subscripts for convenience,

$$
Z=\sum_{u_{1}=0}^{p}\left\{A_{1} B_{1}-E\left(A_{1} B_{1}\right)\right\}=\sum_{u_{1}=0}^{p}\left(\tilde{A}_{1} B_{1}+c_{1} \tilde{B}_{1}\right)
$$


Therefore, independence of $A_{1}$ and $B_{1}$ yields

$$
E(Z \mid g, h)=\sum_{u_{1}=0}^{p} c_{1} \tilde{B}_{1} .
$$

Similarly,

$$
Z^{k}=\prod_{i=1}^{k} \sum_{u_{i}=0}^{p}\left(\tilde{A}_{i} B_{i}+c_{i} \tilde{B}_{i}\right),
$$

so by independence of $A_{i}$ and $B_{j}$, for all $i, j$

$$
\begin{aligned}
E\left(Z^{2} \mid g, h\right)= & \sum_{u_{1}, u_{2}=0}^{p}\left(c_{1} c_{2} \tilde{B}_{1} \tilde{B}_{2}+c_{12} B_{1} B_{2}\right), \\
E\left(Z^{3} \mid g, h\right)= & \sum_{u_{1}, u_{2}, u_{3}=0}^{p}\left(c_{1} c_{2} c_{3} \tilde{B}_{1} \tilde{B}_{2} \tilde{B}_{3}+c_{123} B_{1} B_{2} B_{3}\right. \\
& \left.+c_{12} c_{3} B_{1} B_{2} \tilde{B}_{3}+c_{13} c_{2} B_{1} B_{3} \tilde{B}_{2}+c_{23} c_{1} B_{2} B_{3} \tilde{B}_{1}\right) .
\end{aligned}
$$

Unless otherwise noted, we will use $\sum$ to mean $\sum_{u_{1}, u_{2}, u_{3}, u_{4}=0}^{p}$ for the remainder of the proof. Using (C.19), (C.20), (C.21) in (C.18), we can write $E\left(\prod_{i=1}^{4} Z_{s_{i}}\right)$ as follows: (i) if $s_{i}=t_{i}, i=1, \ldots, 4$,

$$
E\left(Z_{t_{1}} Z_{t_{2}} Z_{t_{3}} Z_{t_{4}}\right)=\sum c_{1} c_{2} c_{3} c_{4} E\left(\tilde{B}_{1} \tilde{B}_{2} \tilde{B}_{3} \tilde{B}_{4}\right)
$$

(ii) if $s_{1}=s_{2}=t_{1}, s_{3}=t_{2}, s_{4}=t_{3}$,

$$
E\left(Z_{t_{1}}^{2} Z_{t_{2}} Z_{t_{3}}\right)=\sum\left\{c_{1} c_{2} c_{3} c_{4} E\left(\tilde{B}_{1} \tilde{B}_{2} \tilde{B}_{3} \tilde{B}_{4}\right)+c_{12} c_{3} c_{4} E\left(B_{1} B_{2} \tilde{B}_{3} \tilde{B}_{4}\right)\right\}
$$

(iii) if $s_{1}=s_{2}=t_{1}, s_{3}=s_{4}=t_{2}$,

$$
E\left(Z_{t_{1}}^{2} Z_{t_{2}}^{2}\right)=\sum\left\{c_{1} c_{2} c_{3} c_{4} E\left(\tilde{B}_{1} \tilde{B}_{2} \tilde{B}_{3} \tilde{B}_{4}\right)+c_{12} c_{34} E\left(B_{1} B_{2} B_{3} B_{4}\right)\right.
$$




$$
\left.+c_{12} c_{3} c_{4} E\left(B_{1} B_{2} \tilde{B}_{3} \tilde{B}_{4}\right)+c_{1} c_{2} c_{34} E\left(\tilde{B}_{1} \tilde{B}_{2} B_{3} B_{4}\right)\right\}
$$

(iv) if $s_{1}=s_{2}=s_{3}=t_{1}, s_{4}=t_{2}$,

$$
\begin{gathered}
E\left(Z_{t_{1}}^{3} Z_{t_{2}}\right)=\sum\left\{c_{1} c_{2} c_{3} c_{4} E\left(\tilde{B}_{1} \tilde{B}_{2} \tilde{B}_{3} \tilde{B}_{4}\right)+c_{123} c_{4} E\left(B_{1} B_{2} B_{3} \tilde{B}_{4}\right)+c_{12} c_{3} c_{4} E\left(B_{1} B_{2} \tilde{B}_{3} \tilde{B}_{4}\right)\right. \\
\left.+c_{13} c_{2} c_{4} E\left(B_{1} B_{3} \tilde{B}_{2} \tilde{B}_{4}\right)+c_{23} c_{1} c_{4} E\left(B_{2} B_{3} \tilde{B}_{1} \tilde{B}_{4}\right)\right\} .
\end{gathered}
$$

We can also write $E\left(Z_{s_{1}} Z_{s_{2}}\right)$ as follows:

(v) if $s_{1}=t_{i}, s_{2}=t_{j}, i \neq j$,

$$
E\left(Z_{t_{i}} Z_{t_{j}}\right)=\sum_{u_{1}, u_{2}=0}^{p} c_{1} c_{2} E\left(\tilde{B}_{1} \tilde{B}_{2}\right)
$$

(vi) if $s_{1}=s_{2}=t_{i}$,

$$
E\left(Z_{t_{i}}^{2}\right)=\sum_{u_{1}, u_{2}=0}^{p}\left\{c_{1} c_{2} E\left(\tilde{B}_{1} \tilde{B}_{2}\right)+c_{12} E\left(B_{1} B_{2}\right)\right\} .
$$

We now proceed to expand $B_{i}$ and $\tilde{B}_{i}$ in terms of $g$ and $h$. Wherever $u_{i}$ and $s_{i}$ are both defined, we use the following notation: $\chi_{i}=\tilde{g}_{s_{i}}^{u_{i}}, \psi_{i}=\tilde{h}_{s_{i}}^{p-u_{i}} ; \chi_{i j}=g_{s_{i}}^{u_{i}+u_{j}}$, $\psi_{i j}=h_{s_{i}}^{2 p-u_{i}-u_{j}} ; \chi_{123}=g_{s_{1}}^{u_{1}+u_{2}+u_{3}}, \psi_{123}=h_{s_{1}}^{3 p-u_{1}-u_{2}-u_{3}} ; \chi_{12,34}=g_{s_{1}}^{u_{1}+u_{2}} g_{s_{3}}^{u_{3}+u_{4}}, \psi_{12,34}=$ $h_{s_{1}}^{2 p-u_{1}-u_{2}} h_{s_{3}}^{2 p-u_{3}-u_{4}}$. Note that

$$
\tilde{B}_{i}=c_{h, i} \chi_{i}+\chi_{i} \psi_{i}+c_{g, i} \psi_{i}
$$

where $c_{h, i}=E\left(h_{s_{i}}^{p-u_{i}}\right), c_{g, i}=E\left(g_{s_{i}}^{u_{i}}\right)$. Four forms of expectations need to be accounted for in (C.22) to (C.25). 
1. $E\left(\tilde{B}_{1} \tilde{B}_{2} \tilde{B}_{3} \tilde{B}_{4}\right)$ will be a linear combination of 81 terms, all of them expectations of products of $\chi_{i}$ and $\psi_{i}, i=1, \ldots, 4$. Denoting by $\langle 1\rangle,\langle 2\rangle,\langle 3\rangle,\langle 4\rangle$ any permutation of $P_{4}$, those terms can be separated into the following categories: terms that vanish due to $E\left(\chi_{i}\right)=E\left(\psi_{i}\right)=0$, namely $E\left(\chi_{\langle 1\rangle} \psi_{\langle 2\rangle} \psi_{\langle 3\rangle} \psi_{\langle 4\rangle}\right), E\left(\psi_{\langle 1\rangle} \chi_{\langle 2\rangle} \chi_{\langle 3\rangle} \chi_{\langle 4\rangle}\right)$, $E\left(\chi_{\langle 1\rangle} \psi_{1} \psi_{2} \psi_{3} \psi_{4}\right)$, and $E\left(\psi_{\langle 1\rangle} \chi_{1} \chi_{2} \chi_{3} \chi_{4}\right)$; non-vanishing terms with four factors, namely $E\left(\chi_{1} \chi_{2} \chi_{3} \chi_{4}\right), E\left(\psi_{1} \psi_{2} \psi_{3} \psi_{4}\right)$, and $E\left(\chi_{\langle 1\rangle} \chi_{\langle 2\rangle} \psi_{\langle 3\rangle} \psi_{\langle 4\rangle}\right)$; non-vanishing terms with five factors, namely $E\left(\chi_{\langle 1\rangle} \chi_{\langle 2\rangle} \psi_{\langle 1\rangle} \psi_{\langle 3\rangle} \psi_{\langle 4\rangle}\right)$ and $E\left(\psi_{\langle 1\rangle} \psi_{\langle 2\rangle} \chi_{\langle 1\rangle} \chi_{\langle 3\rangle} \chi_{\langle 4\rangle}\right)$; terms with six factors, namely $E\left(\chi_{\langle 1\rangle} \chi_{\langle 2\rangle} \psi_{1} \psi_{2} \psi_{3} \psi_{4}\right), E\left(\psi_{\langle 1\rangle} \psi_{\langle 2\rangle} \chi_{1} \chi_{2} \chi_{3} \chi_{4}\right)$, and $E\left(\chi_{\langle 1\rangle} \chi_{\langle 2\rangle} \chi_{\langle 3\rangle} \psi_{\langle 1\rangle} \psi_{\langle 2\rangle} \psi_{\langle 4\rangle}\right)$; terms with seven factors, namely $E\left(\chi_{\langle 1\rangle} \chi_{\langle 2\rangle} \chi_{\langle 3\rangle} \psi_{1} \psi_{2} \psi_{3} \psi_{4}\right)$ and $E\left(\psi_{\langle 1\rangle} \psi_{\langle 2\rangle} \psi_{\langle 3\rangle} \chi_{1} \chi_{2} \chi_{3} \chi_{4}\right)$; a term with eight factors, namely $E\left(\chi_{1} \chi_{2} \chi_{3} \chi_{4} \psi_{1} \psi_{2} \psi_{3} \psi_{4}\right)$. It can be seen from (C.28) that, for each $i=1, \ldots, 4$, the corresponding coefficient will include a factor $c_{h, i}$ if only $\chi_{i}$ is present or $c_{g, i}$ if only $\psi_{i}$ is present.

2. $E\left(B_{1} B_{2} \tilde{B}_{3} \tilde{B}_{4}\right)$ will be a linear combination of 9 terms. Denoting by $\langle 3\rangle,\langle 4\rangle$ any permutation of $\{3,4\}$, these can be grouped in the following categories: $E\left(\chi_{12} \psi_{12} \psi_{3} \psi_{4}\right)$, $E\left(\psi_{12} \chi_{12} \chi_{3} \chi_{4}\right), \quad E\left(\chi_{12} \chi_{\langle 3\rangle} \psi_{12} \psi_{\langle 4\rangle}\right), \quad E\left(\chi_{12} \chi_{\langle 3\rangle} \psi_{12} \psi_{3} \psi_{4}\right), \quad E\left(\psi_{12} \psi_{\langle 3\rangle} \chi_{12} \chi_{3} \chi_{4}\right), \quad$ and $E\left(\chi_{12} \chi_{3} \chi_{4} \psi_{12} \psi_{3} \psi_{4}\right)$. As in the previous case, (C.28) implies that, for each $i=3,4$, the corresponding coefficient will include a factor $c_{h, i}$ if only $\chi_{i}$ is present or $c_{g, i}$ if only $\psi_{i}$ is present.

3. $E\left(B_{1} B_{2} B_{3} \tilde{B}_{4}\right)=c_{h, 4} E\left(\chi_{123} \chi_{4} \psi_{123}\right)+c_{g, 4} E\left(\chi_{123} \psi_{123} \psi_{4}\right)+E\left(\chi_{123} \chi_{4} \psi_{123} \psi_{4}\right)$.

4. $E\left(B_{1} B_{2} B_{3} B_{4}\right)=E\left(\chi_{12,34} \psi_{12,34}\right)$.

In (C.26) and (C.27), the relevant expectations are $E\left(\tilde{B}_{1} \tilde{B}_{2}\right)=c_{h, 1} c_{h, 2} E\left(\chi_{1} \chi_{2}\right)+$ $c_{g, 1} c_{g, 2} E\left(\psi_{1} \psi_{2}\right)+E\left(\chi_{1} \chi_{2} \psi_{1} \psi_{2}\right)$ and $E\left(B_{1} B_{2}\right)=E\left(\chi_{12} \psi_{12}\right)$.

We can now use Theorem 1 to expand each these expectations as $\sum_{q=0}^{\infty} a_{q}$. Let $\mu_{t}$ represent either $\eta_{2 t}$ or $\nu_{2 t}$, with $\gamma_{i j}=E\left(\mu_{t_{i}} \mu_{t_{j}}\right)$, and define $f_{i, t}=f_{i}\left(\mu_{t}\right), f_{i j, t}^{*}=f_{i j}^{*}\left(\mu_{t}\right)$ such that $E\left(f_{i, t}\right)=0$. Denote by $G_{i ; q}, G_{i j ; q}, G_{i j k ; q}, G_{i j ; q}^{*}$ the $q$-th Hermite coefficient of 
$f_{i, t}, f_{i, t} f_{j, t}, f_{i, t} f_{j, t} f_{k, t}, f_{i j, t}^{*}$ respectively.

For $E\left(f_{1, t_{1}} f_{2, t_{2}} f_{3, t_{3}} f_{4, t_{4}}\right)$, we have

$$
\begin{gathered}
a_{q}=\sum_{\substack{v_{\alpha} \geq 0: \\
\Sigma v_{\alpha}=q, \alpha \in Q_{4}}} \prod_{i=1}^{4} G_{i ; w_{i}} \prod_{\alpha \in Q_{4}} \frac{\gamma_{\alpha}^{v_{\alpha}}}{v_{\alpha} !}, \quad w_{k}=\sum_{\alpha \in R_{4, k}} v_{\alpha}, \\
a_{0}=a_{1}=0, \quad a_{2}=G_{1 ; 1} G_{2 ; 1} G_{3 ; 1} G_{4 ; 1}\left(\gamma_{12} \gamma_{34}+\gamma_{13} \gamma_{24}+\gamma_{14} \gamma_{23}\right) .
\end{gathered}
$$

Since $G_{i ; 0}=0$, Theorem 1 yields

$$
\sum_{q=3}^{\infty}\left|a_{q}\right| \leq K \prod_{i=1}^{4}\left(\sum_{\alpha \in R_{4, i}}\left|\gamma_{\alpha}\right|\right)^{\frac{1}{2}} \sum_{\alpha \in Q_{4}}\left|\gamma_{\alpha}\right|
$$

Label the elements of $P_{4}$ as $\langle 1\rangle,\langle 2\rangle,\langle 3\rangle,\langle 4\rangle$, such that $\left|\gamma_{\langle 1\rangle\langle 2\rangle}\right|$ is the largest absolute correlation. Then $\sum_{\alpha \in Q_{4}}\left|\gamma_{\alpha}\right| \leq K\left|\gamma_{\langle 1\rangle\langle 2\rangle}\right|$ and

$$
\prod_{i=1}^{4} \sum_{\alpha \in R_{4, i}}\left|\gamma_{\alpha}\right| \leq K \gamma_{\langle 1\rangle\langle 2\rangle}^{2}\left(\left|\gamma_{\langle 1\rangle\langle 3\rangle}\right|+\left|\gamma_{\langle 2\rangle\langle 3\rangle}\right|+\left|\gamma_{\langle 3\rangle\langle\langle\rangle}\right|\right)\left(\left|\gamma_{\langle 1\rangle\langle 4\rangle}\right|+\left|\gamma_{\langle 2\rangle\langle\langle\rangle}\right|+\left|\gamma_{\langle 3\rangle\langle 4\rangle}\right|\right)
$$

Choosing the second largest absolute correlation, we have a bound of the form

$$
\sum_{q=4}^{\infty}\left|a_{q}\right| \leq K \gamma_{\langle 1\rangle\langle 2\rangle}^{2}\left|\gamma_{\langle 3\rangle\langle 4\rangle}\right| \quad \text { or } \quad \sum_{q=4}^{\infty}\left|a_{q}\right| \leq K \gamma_{\langle 1\rangle\langle 2\rangle}^{2}\left|\gamma_{\langle 1\rangle\langle 3\rangle}\right| .
$$

Therefore, taking all possible permutations for $\langle 1\rangle,\langle 2\rangle,\langle 3\rangle,\langle 4\rangle, \sum_{q=3}^{\infty}\left|a_{q}\right|=O\left(e_{3}\right)$, where $e_{3}=\sum_{\alpha_{1}, \alpha_{2} \in Q_{4}: \alpha_{1} \neq \alpha_{2}} \gamma_{\alpha_{1}}^{2}\left|\gamma_{\alpha_{2}}\right|$, yielding

$$
E\left(f_{1, t_{1}} f_{2, t_{2}} f_{3, t_{3}} f_{4, t_{4}}\right)=\prod_{i=1}^{4} G_{i ; 1}\left(\gamma_{12} \gamma_{34}+\gamma_{13} \gamma_{24}+\gamma_{14} \gamma_{23}\right)+O\left(e_{3}\right)
$$


Again from Theorem 1 but using Corollary 1, defining $e_{2}=\gamma_{12}^{2}+\gamma_{13}^{2}+\gamma_{23}^{2}$,

$$
\begin{aligned}
E\left(f_{1, t_{1}} f_{2, t_{1}} f_{3, t_{2}} f_{4, t_{3}}\right) & =G_{12 ; 0} G_{3 ; 1} G_{4 ; 1} \gamma_{23}+O\left(e_{2}\right), \\
E\left(f_{1, t_{1}} f_{2, t_{1}} f_{3, t_{2}} f_{4, t_{2}}\right) & =G_{12 ; 0} G_{34 ; 0}+O\left(\left|\gamma_{12}\right|\right), \\
E\left(f_{1, t_{1}} f_{2, t_{1}} f_{3, t_{1}} f_{4, t_{2}}\right) & =O\left(\left|\gamma_{12}\right|\right), \\
E\left(f_{1, t_{1}} f_{2, t_{2}} f_{3, t_{3}}\right) & =O\left(e_{2}\right), \\
E\left(f_{1, t_{1}} f_{2, t_{1}} f_{3, t_{2}}\right) & =G_{12 ; 1} G_{3 ; 1} \gamma_{12}+O\left(\gamma_{12}^{2}\right), \\
E\left(f_{1, t_{1}} f_{2, t_{1}} f_{3, t_{1}}\right) & =G_{123 ; 0}, \\
E\left(f_{1, t_{1}} f_{2, t_{2}}\right) & =G_{1 ; 1} G_{2 ; 1} \gamma_{12}+O\left(\gamma_{12}^{2}\right), \\
E\left(f_{1, t_{1}} f_{2, t_{1}}\right) & =G_{12 ; 0}, \\
E\left(f_{12, t_{1}}^{*} f_{3, t_{2}} f_{4, t_{3}}\right) & =G_{12 ; 0}^{*} G_{3 ; 1} G_{4 ; 1} \gamma_{23}+O\left(e_{2}\right), \\
E\left(f_{12, t_{1}}^{*} f_{3, t_{2}} f_{4, t_{2}}\right) & =G_{12 ; 0}^{*} G_{34 ; 0}+O\left(\left|\gamma_{12}\right|\right), \\
E\left(f_{12, t_{1}}^{*} f_{3, t_{2}}\right) & =G_{12 ; 1}^{*} G_{3 ; 1} \gamma_{12}+O\left(\gamma_{12}^{2}\right), \\
E\left(f_{12, t_{1}}^{*}\right) & =G_{12 ; 0}^{*}, \\
E\left(f_{12, t_{1}}^{*} f_{34, t_{2}}^{*}\right) & =G_{12 ; 0}^{*} G_{34 ; 0}^{*}+O\left(\left|\gamma_{12}\right|\right) .
\end{aligned}
$$

Now let the $G$ and $G^{*}$ coefficients in (C.29) to (C.42) apply to the case

$$
f_{i, t}=\tilde{g}_{t}^{u_{i}}, \quad f_{i j, t}^{*}=g_{t}^{u_{i}+u_{j}}
$$

while corresponding $G^{\prime}$ and $G^{* \prime}$ coefficients apply to

$$
f_{i, t}=\tilde{h}_{t}^{p-u_{i}}, \quad f_{i j, t}^{*}=h_{t}^{2 p-u_{i}-u_{j}}
$$


We can approximate each term in the expansion of (C.22) to (C.27) using (C.29) to (C.42):

(i) $E\left(Z_{t_{1}} Z_{t_{2}} Z_{t_{3}} Z_{t_{4}}\right)$. Denote by $\langle 1\rangle,\langle 2\rangle,\langle 3\rangle,\langle 4\rangle$ any permutation of $P_{4}$. Using (C.29), (C.33), (C.36), the only terms that are not $O\left(e_{3}\right)$ are:

$$
\begin{aligned}
E\left(\chi_{1} \chi_{2} \chi_{3} \chi_{4}\right) & =G_{1 ; 1} G_{2 ; 1} G_{3 ; 1} G_{4 ; 1}\left(\rho_{12} \rho_{34}+\rho_{13} \rho_{24}+\rho_{14} \rho_{23}\right)+O\left(e_{3}\right), \\
E\left(\psi_{1} \psi_{2} \psi_{3} \psi_{4}\right) & =G_{1 ; 1}^{\prime} G_{2 ; 1}^{\prime} G_{3 ; 1}^{\prime} G_{4 ; 1}^{\prime}\left(\rho_{12}^{\prime} \rho_{34}^{\prime}+\rho_{13}^{\prime} \rho_{24}^{\prime}+\rho_{14}^{\prime} \rho_{23}^{\prime}\right)+O\left(e_{3}\right), \\
E\left(\chi_{\langle 1\rangle} \chi_{\langle 2\rangle}\right) E\left(\psi_{\langle 3\rangle} \psi_{\langle 4\rangle}\right) & =G_{\langle 1\rangle ; 1} G_{\langle 2\rangle ; 1} G_{\langle 3\rangle ; 1}^{\prime} G_{\langle 4\rangle ; 1}^{\prime} \rho_{\langle 1\rangle\langle 2\rangle} \rho_{\langle 3\rangle\langle 4\rangle}^{\prime}+O\left(e_{3}\right) .
\end{aligned}
$$

(ii) $E\left(Z_{t_{1}}^{2} Z_{t_{2}} Z_{t_{3}}\right)$. Using (C.30), (C.33), (C.34), (C.36), (C.37), the only terms in $E\left(\tilde{B}_{1} \tilde{B}_{2} \tilde{B}_{3} \tilde{B}_{4}\right)$ that are not $O\left(e_{2}\right)$ are:

$$
\begin{aligned}
E\left(\chi_{1} \chi_{2} \chi_{3} \chi_{4}\right) & =G_{12 ; 0} G_{3 ; 1} G_{4 ; 1} \rho_{23}+O\left(e_{2}\right), \\
E\left(\psi_{1} \psi_{2} \psi_{3} \psi_{4}\right) & =G_{12 ; 0}^{\prime} G_{3 ; 1}^{\prime} G_{4 ; 1}^{\prime} \rho_{23}^{\prime}+O\left(e_{2}\right), \\
E\left(\chi_{1} \chi_{2}\right) E\left(\psi_{3} \psi_{4}\right) & =G_{12 ; 0} G_{3 ; 1}^{\prime} G_{4 ; 1}^{\prime} \rho_{23}^{\prime}+O\left(e_{2}\right), \\
E\left(\psi_{1} \psi_{2}\right) E\left(\chi_{3} \chi_{4}\right) & =G_{12 ; 0}^{\prime} G_{3 ; 1} G_{4 ; 1} \rho_{23}+O\left(e_{2}\right), \\
E\left(\chi_{1} \chi_{2}\right) E\left(\psi_{1} \psi_{2} \psi_{3} \psi_{4}\right) & =G_{12 ; 0} G_{12 ; 0}^{\prime} G_{3 ; 1}^{\prime} G_{4 ; 1}^{\prime} \rho_{23}^{\prime}+O\left(e_{2}\right), \\
E\left(\psi_{1} \psi_{2}\right) E\left(\chi_{1} \chi_{2} \chi_{3} \chi_{4}\right) & =G_{12 ; 0}^{\prime} G_{12 ; 0} G_{3 ; 1} G_{4 ; 1} \rho_{23}+O\left(e_{2}\right) .
\end{aligned}
$$

Using (C.38), (C.40), (C.41) the only terms in $E\left(B_{1} B_{2} \tilde{B}_{3} \tilde{B}_{4}\right)$ that are not $O\left(e_{2}\right)$ are:

$$
\begin{aligned}
& E\left(\chi_{12}\right) E\left(\psi_{12} \psi_{3} \psi_{4}\right)=G_{12,0}^{*} G_{12 ; 0}^{* \prime} G_{3 ; 1}^{\prime} G_{4 ; 1}^{\prime} \rho_{23}^{\prime}+O\left(e_{2}\right), \\
& E\left(\psi_{12}\right) E\left(\chi_{12} \chi_{3} \chi_{4}\right)=G_{12,0}^{* \prime} G_{12 ; 0}^{*} G_{3 ; 1} G_{4 ; 1} \rho_{23}+O\left(e_{2}\right) .
\end{aligned}
$$


(iii) $E\left(Z_{t_{1}}^{2} Z_{t_{2}}^{2}\right)$. From (C.31), (C.34), (C.36), (C.37) it follows that all terms in $E\left(\tilde{B}_{1} \tilde{B}_{2} \tilde{B}_{3} \tilde{B}_{4}\right)$ will be $O\left(\left|\rho_{12}\right|\right)$ except the ones only involving (C.31) and (C.37):

$$
\begin{aligned}
E\left(\chi_{1} \chi_{2} \chi_{3} \chi_{4}\right) & =G_{12 ; 0} G_{34 ; 0}+O\left(\left|\rho_{12}\right|\right) \\
E\left(\psi_{1} \psi_{2} \psi_{3} \psi_{4}\right) & =G_{12 ; 0}^{\prime} G_{34 ; 0}^{\prime}+O\left(\left|\rho_{12}\right|\right) \\
E\left(\chi_{1} \chi_{2}\right) E\left(\psi_{3} \psi_{4}\right) & =G_{12 ; 0} G_{34 ; 0}^{\prime} \\
E\left(\psi_{1} \psi_{2}\right) E\left(\chi_{3} \chi_{4}\right) & =G_{12 ; 0}^{\prime} G_{34 ; 0} \\
E\left(\chi_{1} \chi_{2}\right) E\left(\psi_{1} \psi_{2} \psi_{3} \psi_{4}\right) & =G_{12 ; 0} G_{12 ; 0}^{\prime} G_{34 ; 0}^{\prime}+O\left(\left|\rho_{12}\right|\right) \\
E\left(\chi_{3} \chi_{4}\right) E\left(\psi_{1} \psi_{2} \psi_{3} \psi_{4}\right) & =G_{34 ; 0} G_{12 ; 0}^{\prime} G_{34 ; 0}^{\prime}+O\left(\left|\rho_{12}\right|\right) \\
E\left(\psi_{1} \psi_{2}\right) E\left(\chi_{1} \chi_{2} \chi_{3} \chi_{4}\right) & =G_{12 ; 0}^{\prime} G_{12 ; 0} G_{34 ; 0}+O\left(\left|\rho_{12}\right|\right) \\
E\left(\psi_{3} \psi_{4}\right) E\left(\chi_{1} \chi_{2} \chi_{3} \chi_{4}\right) & =G_{34 ; 0}^{\prime} G_{12 ; 0} G_{34 ; 0}+O\left(\left|\rho_{12}\right|\right) \\
E\left(\chi_{1} \chi_{2} \chi_{3} \chi_{4}\right) E\left(\psi_{1} \psi_{2} \psi_{3} \psi_{4}\right) & =G_{12 ; 0} G_{34 ; 0} G_{12 ; 0}^{\prime} G_{34 ; 0}^{\prime}+O\left(\left|\rho_{12}\right|\right) .
\end{aligned}
$$

Similarly, from (C.39), (C.40), (C.41), (C.42), all terms in $E\left(B_{1} B_{2} \tilde{B}_{3} \tilde{B}_{4}\right)$, $E\left(\tilde{B}_{1} \tilde{B}_{2} B_{3} B_{4}\right)$, and $E\left(B_{1} B_{2} B_{3} B_{4}\right)$ will be $O\left(\left|\rho_{12}\right|\right)$ except the following:

$$
\begin{aligned}
E\left(\chi_{12}\right) E\left(\psi_{12} \psi_{3} \psi_{4}\right) & =G_{12 ; 0}^{*} G_{12 ; 0}^{* \prime} G_{34 ; 0}^{\prime}+O\left(\left|\rho_{12}\right|\right), \\
E\left(\chi_{34}\right) E\left(\psi_{34} \psi_{1} \psi_{2}\right) & =G_{12 ; 0}^{*} G_{12 ; 0}^{* \prime} G_{34 ; 0}^{\prime}+O\left(\left|\rho_{12}\right|\right), \\
E\left(\psi_{12}\right) E\left(\chi_{12} \chi_{3} \chi_{4}\right) & =G_{12 ; 0}^{* \prime} G_{12 ; 0}^{*} G_{34 ; 0}+O\left(\left|\rho_{12}\right|\right), \\
E\left(\psi_{34}\right) E\left(\chi_{34} \chi_{1} \chi_{2}\right) & =G_{12 ; 0}^{* \prime} G_{12 ; 0}^{*} G_{34 ; 0}+O\left(\left|\rho_{12}\right|\right), \\
E\left(\chi_{12} \chi_{3} \chi_{4}\right) E\left(\psi_{12} \psi_{3} \psi_{4}\right) & =G_{12 ; 0}^{*} G_{34 ; 0} G_{12 ; 0}^{* \prime} G_{34 ; 0}^{\prime}+O\left(\left|\rho_{12}\right|\right), \\
E\left(\chi_{34} \chi_{1} \chi_{2}\right) E\left(\psi_{34} \psi_{1} \psi_{2}\right) & =G_{12 ; 0}^{*} G_{34 ; 0} G_{12 ; 0}^{* \prime} G_{34 ; 0}^{\prime}+O\left(\left|\rho_{12}\right|\right), \\
E\left(\chi_{12,34}\right) E\left(\psi_{12,34}\right) & =G_{12 ; 0}^{*} G_{34 ; 0}^{*} G_{12 ; 0}^{* \prime} G_{34 ; 0}^{* \prime}+O\left(\left|\rho_{12}\right|\right) .
\end{aligned}
$$


(iv) $E\left(Z_{t_{1}}^{3} Z_{t_{2}}\right)$. Using (C.32), (C.34), (C.35), (C.36), (C.37) in $E\left(\tilde{B}_{1} \tilde{B}_{2} \tilde{B}_{3} \tilde{B}_{4}\right)$ note that at least one factor in each term necessarily involves $t_{2}$. Therefore, one of (C.32), (C.34), (C.36) will apply, making all terms $O\left(\left|\rho_{12}\right|\right)$.

Similarly, in $E\left(B_{1} B_{2} \tilde{B}_{3} \tilde{B}_{4}\right), E\left(B_{1} \tilde{B}_{2} B_{3} \tilde{B}_{4}\right), E\left(\tilde{B}_{1} B_{2} B_{3} \tilde{B}_{4}\right)$, and $E\left(B_{1} B_{2} B_{3} \tilde{B}_{4}\right)$, at least one factor in each term necessarily involves $t_{2}$. Thus, (C.40) will apply for some function $f_{i j, t}^{*}$, not necessarily one given in (C.43) or (C.44), making all terms $O\left(\left|\rho_{12}\right|\right)$.

(v) $E\left(Z_{t_{i}} Z_{t_{j}}\right)$. Using (C.36), the following are not $O\left(\rho_{i j}^{2}\right)$ :

$$
E\left(\chi_{1} \chi_{2}\right)=G_{1 ; 1} G_{2 ; 1} \rho_{i j}+O\left(\rho_{i j}^{2}\right), \quad E\left(\psi_{1} \psi_{2}\right)=G_{1 ; 1}^{\prime} G_{2 ; 1}^{\prime} \rho_{i j}^{\prime}+O\left(\rho_{i j}^{2}\right) .
$$

(vi) $E\left(Z_{t_{i}}^{2}\right)$. Using (C.37), $E\left(\tilde{B}_{1} \tilde{B}_{2}\right)$ and $E\left(B_{1} B_{2}\right)$ include the following terms:

$$
\begin{aligned}
& E\left(\chi_{1} \chi_{2}\right)=G_{12 ; 0}, \quad E\left(\chi_{1} \chi_{2}\right) E\left(\psi_{1} \psi_{2}\right)=G_{12 ; 0} G_{12 ; 0}^{\prime}, \\
& E\left(\psi_{1} \psi_{2}\right)=G_{12 ; 0}^{\prime}, \quad E\left(\chi_{12}\right) E\left(\psi_{12}\right)=G_{12 ; 0}^{*} G_{12 ; 0}^{* \prime} .
\end{aligned}
$$

We now compute the coefficients of the leading terms listed above. Define

$$
\begin{array}{rlll}
L_{i}=c_{i} c_{h, i} G_{i ; 1}, & \bar{L}_{1}=\sum_{u_{i}=0}^{p} L_{i}, & L_{i}^{\prime}=c_{i} c_{g, i} G_{i ; 1}^{\prime}, & \bar{L}_{1}^{\prime}=\sum_{u_{i}=0}^{p} L_{i}^{\prime} ; \\
L_{i j}=c_{i} c_{h, i} c_{j} c_{h, j} G_{i j ; 0}, & \bar{L}_{2}=\sum_{u_{i}, u_{j}=0}^{p} L_{i j}, & L_{i j}^{\prime}=c_{i} c_{g, i} c_{j} c_{g, j} G_{i j ; 0}^{\prime}, & \bar{L}_{2}^{\prime}=\sum_{u_{i}, u_{j}=0}^{p} L_{i j}^{\prime} ; \\
L_{i j}^{*}=c_{i} c_{j} G_{i j ; 0} G_{i j ; 0}^{\prime}, & \bar{L}_{2}^{*}=\sum_{u_{i}, u_{j}=0}^{p} L_{i j}^{*}, & L_{i j}^{* *}=c_{i j} G_{i j ; 0}^{*} G_{i j ; 0}^{* \prime}, & \bar{L}_{2}^{* *}=\sum_{u_{i}, u_{j}=0}^{p} L_{i j}^{* *} .
\end{array}
$$

Note that $L_{p}=c_{p} c_{h, p} G_{p ; 1}=\beta_{2}^{p} E\left(\eta_{1 t}^{p}\right) E\left\{g^{p}\left(\eta_{2 t}\right) H_{1}\left(\eta_{2 t}\right)\right\} \neq 0$ by assumption, but $L_{i}=0$ for any $i<p$. Hence $\bar{L}_{1}=L_{p} \neq 0$. The contributions of the non-negligible terms will be: 
(i) $E\left(Z_{t_{1}} Z_{t_{2}} Z_{t_{3}} Z_{t_{4}}\right)$

$$
\begin{aligned}
E\left(\chi_{1} \chi_{2} \chi_{3} \chi_{4}\right) & : L_{1} L_{2} L_{3} L_{4}\left(\rho_{12} \rho_{34}+\rho_{13} \rho_{24}+\rho_{14} \rho_{23}\right)+O\left(e_{3}\right) ; \\
E\left(\psi_{1} \psi_{2} \psi_{3} \psi_{4}\right) & : L_{1}^{\prime} L_{2}^{\prime} L_{3}^{\prime} L_{4}^{\prime}\left(\rho_{12}^{\prime} \rho_{34}^{\prime}+\rho_{13}^{\prime} \rho_{24}^{\prime}+\rho_{14}^{\prime} \rho_{23}^{\prime}\right)+O\left(e_{3}\right) ; \\
E\left(\chi_{\langle 1\rangle} \chi_{\langle 2\rangle}\right) E\left(\psi_{\langle 3\rangle} \psi_{\langle 4\rangle}\right) & : L_{\langle 1\rangle} L_{\langle 2\rangle} L_{\langle 3\rangle}^{\prime} L_{\langle 4\rangle}^{\prime} \rho_{\langle 1\rangle\langle 2\rangle} \rho_{\langle 3\rangle\langle\langle\rangle}^{\prime}+O\left(e_{3}\right) .
\end{aligned}
$$

Thus, $E\left(Z_{t_{1}} Z_{t_{2}} Z_{t_{3}} Z_{t_{4}}\right)$ is

$$
\begin{aligned}
& \sum\left\{L_{1} L_{2} L_{3} L_{4}\left(\rho_{12} \rho_{34}+\rho_{13} \rho_{24}+\rho_{14} \rho_{23}\right)+L_{1}^{\prime} L_{2}^{\prime} L_{3}^{\prime} L_{4}^{\prime}\left(\rho_{12}^{\prime} \rho_{34}^{\prime}+\rho_{13}^{\prime} \rho_{24}^{\prime}+\rho_{14}^{\prime} \rho_{23}^{\prime}\right)\right. \\
& +L_{1} L_{2} L_{3}^{\prime} L_{4}^{\prime} \rho_{12} \rho_{34}^{\prime}+L_{1}^{\prime} L_{2}^{\prime} L_{3} L_{4} \rho_{12}^{\prime} \rho_{34}+L_{1} L_{2}^{\prime} L_{3} L_{4}^{\prime} \rho_{13} \rho_{24}^{\prime}+L_{1}^{\prime} L_{2} L_{3}^{\prime} L_{4} \rho_{13}^{\prime} \rho_{24} \\
& \left.+L_{1} L_{2}^{\prime} L_{3}^{\prime} L_{4} \rho_{14} \rho_{23}^{\prime}+L_{1}^{\prime} L_{2} L_{3} L_{4}^{\prime} \rho_{14}^{\prime} \rho_{23}\right\}+O\left(e_{3}\right) \\
= & \sum\left\{\left(L_{1} L_{2} \rho_{12}+L_{1}^{\prime} L_{2}^{\prime} \rho_{12}^{\prime}\right)\left(L_{3} L_{4} \rho_{34}+L_{3}^{\prime} L_{4}^{\prime} \rho_{34}^{\prime}\right)\right. \\
& +\left(L_{1} L_{3} \rho_{13}+L_{1}^{\prime} L_{3}^{\prime} \rho_{13}^{\prime}\right)\left(L_{2} L_{4} \rho_{24}+L_{2}^{\prime} L_{4}^{\prime} \rho_{24}^{\prime}\right) \\
& \left.+\left(L_{1} L_{4} \rho_{14}+L_{1}^{\prime} L_{4}^{\prime} \rho_{14}^{\prime}\right)\left(L_{2} L_{3} \rho_{23}+L_{2}^{\prime} L_{3}^{\prime} \rho_{23}^{\prime}\right)\right\}+O\left(e_{3}\right) \\
= & \left(\bar{L}_{1}^{2} \rho_{12}+\bar{L}_{1}^{\prime 2} \rho_{12}^{\prime}\right)\left(\bar{L}_{1}^{2} \rho_{34}+\bar{L}_{1}^{\prime 2} \rho_{34}^{\prime}\right)+\left(\bar{L}_{1}^{2} \rho_{13}+\bar{L}_{1}^{\prime 2} \rho_{13}^{\prime}\right)\left(\bar{L}_{1}^{2} \rho_{24}+\bar{L}_{1}^{\prime 2} \rho_{24}^{\prime}\right) \\
& +\left(\bar{L}_{1}^{2} \rho_{14}+\bar{L}_{1}^{\prime 2} \rho_{14}^{\prime}\right)\left(\bar{L}_{1}^{2} \rho_{23}+\bar{L}_{1}^{\prime 2} \rho_{23}^{\prime}\right)+O\left(e_{3}\right) .
\end{aligned}
$$

(ii) $E\left(Z_{t_{1}}^{2} Z_{t_{2}} Z_{t_{3}}\right)$

$$
\begin{array}{rr}
E\left(\chi_{1} \chi_{2} \chi_{3} \chi_{4}\right): L_{12} L_{3} L_{4} \rho_{23}+O\left(e_{2}\right) ; & E\left(\chi_{1} \chi_{2}\right) E\left(\psi_{1} \psi_{2} \psi_{3} \psi_{4}\right): L_{12}^{*} L_{3}^{\prime} L_{4}^{\prime} \rho_{23}^{\prime}+O\left(e_{2}\right) ; \\
E\left(\psi_{1} \psi_{2} \psi_{3} \psi_{4}\right): L_{12}^{\prime} L_{3}^{\prime} L_{4}^{\prime} \rho_{23}^{\prime}+O\left(e_{2}\right) ; & E\left(\psi_{1} \psi_{2}\right) E\left(\chi_{1} \chi_{2} \chi_{3} \chi_{4}\right): L_{12}^{*} L_{3} L_{4} \rho_{23}+O\left(e_{2}\right) ; \\
E\left(\chi_{1} \chi_{2}\right) E\left(\psi_{3} \psi_{4}\right): L_{12} L_{3}^{\prime} L_{4}^{\prime} \rho_{23}^{\prime}+O\left(e_{2}\right) ; & E\left(\chi_{12}\right) E\left(\psi_{12} \psi_{3} \psi_{4}\right): L_{12}^{* *} L_{3}^{\prime} L_{4}^{\prime} \rho_{23}^{\prime}+O\left(e_{2}\right) ; \\
E\left(\psi_{1} \psi_{2}\right) E\left(\chi_{3} \chi_{4}\right): L_{12}^{\prime} L_{3} L_{4} \rho_{23}+O\left(e_{2}\right) ; & E\left(\psi_{12}\right) E\left(\chi_{12} \chi_{3} \chi_{4}\right): L_{12}^{* *} L_{3} L_{4} \rho_{23}+O\left(e_{2}\right) .
\end{array}
$$


Thus,

$$
\begin{aligned}
E\left(Z_{t_{1}}^{2} Z_{t_{2}} Z_{t_{3}}\right)= & \sum\left(L_{12} L_{3} L_{4} \rho_{23}+L_{12}^{\prime} L_{3}^{\prime} L_{4}^{\prime} \rho_{23}^{\prime}+L_{12} L_{3}^{\prime} L_{4}^{\prime} \rho_{23}^{\prime}+L_{12}^{\prime} L_{3} L_{4} \rho_{23}\right. \\
& \left.+L_{12}^{*} L_{3}^{\prime} L_{4}^{\prime} \rho_{23}^{\prime}+L_{12}^{*} L_{3} L_{4} \rho_{23}+L_{12}^{* *} L_{3}^{\prime} L_{4}^{\prime} \rho_{23}^{\prime}+L_{12}^{* *} L_{3} L_{4} \rho_{23}\right)+O\left(e_{2}\right) \\
= & \sum\left(L_{12}+L_{12}^{\prime}+L_{12}^{*}+L_{12}^{* *}\right)\left(L_{3} L_{4} \rho_{23}+L_{3}^{\prime} L_{4}^{\prime} \rho_{23}^{\prime}\right)+O\left(e_{2}\right) \\
= & \left(\bar{L}_{2}+\bar{L}_{2}^{\prime}+\bar{L}_{2}^{*}+\bar{L}_{2}^{* *}\right)\left(\bar{L}_{1}^{2} \rho_{23}+\bar{L}_{1}^{\prime 2} \rho_{23}^{\prime}\right)+O\left(e_{2}\right) .
\end{aligned}
$$

(iii) $E\left(Z_{t_{1}}^{2} Z_{t_{2}}^{2}\right)$

$$
\begin{array}{rr}
E\left(\chi_{1} \chi_{2} \chi_{3} \chi_{4}\right): L_{12} L_{34}+O\left(\left|\rho_{12}\right|\right) ; & E\left(\chi_{1} \chi_{2}\right) E\left(\psi_{1} \psi_{2} \psi_{3} \psi_{4}\right): L_{12}^{*} L_{34}^{\prime}+O\left(\left|\rho_{12}\right|\right) ; \\
E\left(\psi_{1} \psi_{2} \psi_{3} \psi_{4}\right): L_{12}^{\prime} L_{34}^{\prime}+O\left(\left|\rho_{12}\right|\right) ; & E\left(\chi_{3} \chi_{4}\right) E\left(\psi_{1} \psi_{2} \psi_{3} \psi_{4}\right): L_{12}^{\prime} L_{34}^{*}+O\left(\left|\rho_{12}\right|\right) ; \\
E\left(\chi_{1} \chi_{2}\right) E\left(\psi_{3} \psi_{4}\right): L_{12} L_{34}^{\prime} ; & E\left(\psi_{1} \psi_{2}\right) E\left(\chi_{1} \chi_{2} \chi_{3} \chi_{4}\right): L_{12}^{*} L_{34}+O\left(\left|\rho_{12}\right|\right) ; \\
E\left(\psi_{1} \psi_{2}\right) E\left(\chi_{3} \chi_{4}\right): L_{12}^{\prime} L_{34} ; & E\left(\psi_{3} \psi_{4}\right) E\left(\chi_{1} \chi_{2} \chi_{3} \chi_{4}\right): L_{12} L_{34}^{*}+O\left(\left|\rho_{12}\right|\right) ; \\
E\left(\chi_{12}\right) E\left(\psi_{12} \psi_{3} \psi_{4}\right): L_{12}^{* *} L_{34}^{\prime}+O\left(\left|\rho_{12}\right|\right) ; & E\left(\chi_{12} \chi_{3} \chi_{4}\right) E\left(\psi_{12} \psi_{3} \psi_{4}\right): L_{12}^{* *} L_{34}^{*}+O\left(\left|\rho_{12}\right|\right) ; \\
E\left(\chi_{34}\right) E\left(\psi_{34} \psi_{1} \psi_{2}\right): L_{12}^{\prime} L_{34}^{* *}+O\left(\left|\rho_{12}\right|\right) ; & E\left(\chi_{34} \chi_{1} \chi_{2}\right) E\left(\psi_{34} \psi_{1} \psi_{2}\right): L_{12}^{*} L_{34}^{* *}+O\left(\left|\rho_{12}\right|\right) ; \\
E\left(\psi_{12}\right) E\left(\chi_{12} \chi_{3} \chi_{4}\right): L_{12}^{* *} L_{34}+O\left(\left|\rho_{12}\right|\right) ; & E\left(\chi_{1} \chi_{2} \chi_{3} \chi_{4}\right) E\left(\psi_{1} \psi_{2} \psi_{3} \psi_{4}\right): L_{12}^{*} L_{34}^{*}+O\left(\left|\rho_{12}\right|\right) ; \\
E\left(\psi_{34}\right) E\left(\chi_{34} \chi_{1} \chi_{2}\right): L_{12} L_{34}^{* *}+O\left(\left|\rho_{12}\right|\right) ; & E\left(\chi_{12,34}\right) E\left(\psi_{12,34}\right): L_{12}^{* *} L_{34}^{* *}+O\left(\left|\rho_{12}\right|\right) .
\end{array}
$$

Thus,

$$
\begin{aligned}
E\left(Z_{t_{1}}^{2} Z_{t_{2}}^{2}\right)= & \sum\left(L_{12} L_{34}+L_{12}^{\prime} L_{34}^{\prime}+L_{12} L_{34}^{\prime}+L_{12}^{\prime} L_{34}+L_{12}^{*} L_{34}^{\prime}\right. \\
& +L_{12}^{\prime} L_{34}^{*}+L_{12}^{*} L_{34}+L_{12} L_{34}^{*}+L_{12}^{*} L_{34}^{*}+L_{12}^{* *} L_{34}^{\prime}+L_{12}^{* *} L_{34} \\
& \left.+L_{12}^{* *} L_{34}^{*}+L_{12}^{\prime} L_{34}^{* *}+L_{12} L_{34}^{* *}+L_{12}^{*} L_{34}^{* *}+L_{12}^{* *} L_{34}^{* *}\right)+O\left(\left|\rho_{12}\right|\right) \\
= & \sum\left(L_{12}+L_{12}^{\prime}+L_{12}^{*}+L_{12}^{* *}\right)\left(L_{34}+L_{34}^{\prime}+L_{34}^{*}+L_{34}^{* *}\right)+O\left(\left|\rho_{12}\right|\right) \\
= & \left(\bar{L}_{2}+\bar{L}_{2}^{\prime}+\bar{L}_{2}^{*}+\bar{L}_{2}^{* *}\right)^{2}+O\left(\left|\rho_{12}\right|\right) .
\end{aligned}
$$


(iv) $E\left(Z_{t_{1}}^{3} Z_{t_{2}}\right)$

$$
E\left(Z_{t_{1}}^{3} Z_{t_{2}}\right)=O\left(\left|\rho_{12}\right|\right)
$$

(v) $E\left(Z_{t_{i}} Z_{t_{j}}\right), i \neq j$

$$
E\left(\chi_{1} \chi_{2}\right): L_{1} L_{2} \rho_{i j}+O\left(\rho_{i j}^{2}\right) ; \quad E\left(\psi_{1} \psi_{2}\right): L_{1}^{\prime} L_{2}^{\prime} \rho_{i j}^{\prime}+O\left(\rho_{i j}^{2}\right)
$$

Thus,

$$
E\left(Z_{t_{i}} Z_{t_{j}}\right)=\sum_{u_{1}, u_{2}=0}^{p}\left(L_{1} L_{2} \rho_{i j}+L_{1}^{\prime} L_{2}^{\prime} \rho_{i j}^{\prime}\right)+O\left(\rho_{i j}^{2}\right)=\bar{L}_{1}^{2} \rho_{i j}+\bar{L}_{1}^{\prime 2} \rho_{i j}^{\prime}+O\left(\rho_{i j}^{2}\right) .
$$

(vi) $E\left(Z_{t_{i}}^{2}\right)$

$$
\begin{array}{lr}
E\left(\chi_{1} \chi_{2}\right): L_{12} ; & E\left(\chi_{1} \chi_{2}\right) E\left(\psi_{1} \psi_{2}\right): L_{12}^{*} ; \\
E\left(\psi_{1} \psi_{2}\right): L_{12}^{\prime} ; & E\left(\chi_{12}\right) E\left(\psi_{12}\right): L_{12}^{* *} .
\end{array}
$$

Thus,

$$
E\left(Z_{t_{i}}^{2}\right)=\sum_{u_{1}, u_{2}=0}^{p}\left(L_{12}+L_{12}^{\prime}+L_{12}^{*}+L_{12}^{* *}\right)=\bar{L}_{2}+\bar{L}_{2}^{\prime}+\bar{L}_{2}^{*}+\bar{L}_{2}^{* *} .
$$

Define $V_{t}$ as a mean-zero Gaussian $I(d)$ process with $E\left(V_{t}^{2}\right)=\bar{L}_{2}+\bar{L}_{2}^{\prime}+\bar{L}_{2}^{*}+\bar{L}_{2}^{* *}$ and $E\left(V_{t_{i}} V_{t_{j}}\right)=\bar{L}_{1}^{2} \rho_{i j}+\bar{L}_{1}^{\prime 2} \rho_{i j}^{\prime}$, for $i \neq j$. Using equations (C.45) to (C.50) to compute the covariances of interest in each case, they are easily shown to be identical to

$$
\operatorname{Cov}\left(V_{s_{1}} V_{s_{2}}, V_{s_{3}} V_{s_{4}}\right)=E\left(V_{s_{1}} V_{s_{3}}\right) E\left(V_{s_{2}} V_{s_{4}}\right)+E\left(V_{s_{1}} V_{s_{4}}\right) E\left(V_{s_{2}} V_{s_{3}}\right)
$$


up to the desired approximation errors.

Lemma 7 If $V_{t}$ is Gaussian I(1/4), under (4.4), $\operatorname{Var}\left\{\hat{F}_{V V}\left(\lambda_{m}\right)\right\}=O\left(n^{-1} \log m\right)$.

Proof. Let $\rho_{j}=\operatorname{Cov}\left(V_{0}, V_{j}\right)$ and assume $\rho_{0}=1$, without loss of generality. By assumption, $\left|\rho_{j}\right| \leq K j^{-1 / 2}$. We will use similar methods to the proof of Lemma 10 in Robinson (1994b), including the decomposition

$$
\begin{aligned}
\operatorname{Var}\left\{\hat{F}_{V V}\left(\lambda_{m}\right)\right\} & =\frac{1}{n^{4}} \sum_{s, t, u, v=1}^{n} \operatorname{Cov}\left(V_{s} V_{t}, V_{u} V_{v}\right) D_{m}\left(\lambda_{t-s}\right) \overline{D_{m}\left(\lambda_{v-u}\right)} \\
& =\frac{1}{n^{4}} \sum_{s, t, u, v=1}^{n}\left(\rho_{u-s} \rho_{v-t}+\rho_{v-s} \rho_{u-t}\right) D_{m}\left(\lambda_{t-s}\right) \overline{D_{m}\left(\lambda_{v-u}\right)} \\
& =\frac{1}{n^{4}} \sum_{j, k=1}^{m}\left(W_{j, k-j} W_{k, j-k}+W_{j,-j-k} W_{-k, j+k}\right),
\end{aligned}
$$

where $W_{j, k}=\sum_{u=1-n}^{n-1} \rho_{u} e^{i \lambda_{j} u} T_{k}(u)$ and $T_{k}(u)=\sum_{t=1+u^{+}}^{n-u^{-}} e^{i t \lambda_{k}}$, denoting the positive and negative parts of $u$ by $u^{+}=(|u|+u) / 2$ and $u^{-}=(|u|-u) / 2$ respectively. Note that Robinson (1994b) has a typo in this decomposition, using $k$ instead of $-k$ in the first index of the last $W$. However, the correct expression is used in the remainder of his proof.

To bound $W_{j, 0}$, for $j=1, \ldots, m$, note that $T_{0}(u)=n-|u|$. Summation by parts gives

$$
\begin{aligned}
W_{j, 0} & =\sum_{u=1-n}^{n-1}(n-|u|) \rho_{u} e^{i \lambda_{j} u}=n+\sum_{u=1}^{n-1}(n-u) \rho_{u}\left(e^{i \lambda_{j} u}+e^{-i \lambda_{j} u}\right) \\
& =n+\sum_{u=1}^{n-1}\left\{(n-u) \rho_{u}-(n-u-1) \rho_{u+1}\right\}\left\{D_{u}\left(\lambda_{j}\right)+\overline{D_{u}\left(\lambda_{j}\right)}\right\},
\end{aligned}
$$


so using (C.3) we get

$$
\begin{aligned}
\left|W_{j, 0}\right| & \leq n+K \sum_{u=1}^{n-1}\left\{(n-u)\left|\rho_{u}-\rho_{u+1}\right|+\left|\rho_{u+1}\right|\right\}\left|D_{u}\left(\lambda_{j}\right)\right| \\
& \leq n+K \sum_{u=1}^{n-1} \frac{n}{u}\left|\rho_{u+1}\right|\left|D_{u}\left(\lambda_{j}\right)\right| \leq n+K n \sum_{u=1}^{[n / j]} u^{-\frac{1}{2}}+K \frac{n^{2}}{j} \sum_{u=[n / j]}^{n} u^{-\frac{3}{2}} \\
& \leq n+K n\left(\frac{n}{j}\right)^{\frac{1}{2}}+K \frac{n^{2}}{j}\left(\frac{n}{j}\right)^{-\frac{1}{2}} \leq K \frac{n^{\frac{3}{2}}}{j^{\frac{1}{2}}}
\end{aligned}
$$

For $k \neq 0$ and $u>0,\left(\right.$ B.1) implies that $T_{k}(0)=0$,

$$
\begin{aligned}
T_{k}(u) & =\sum_{t=1+u}^{n} e^{i t \lambda_{k}}=\sum_{t=1}^{n} e^{i t \lambda_{k}}-\sum_{t=1}^{u} e^{i t \lambda_{k}}=-D_{u}\left(\lambda_{k}\right), \\
T_{k}(-u) & =\sum_{t=1}^{n-u} e^{i t \lambda_{k}}=\sum_{t=1}^{n} e^{i t \lambda_{k}}-e^{i \lambda_{k}} \sum_{t=n-u}^{n-1} e^{i t \lambda_{k}}=-e^{i \lambda_{k}} \overline{D_{u}\left(\lambda_{k}\right)} .
\end{aligned}
$$

Therefore, using summation by parts,

$$
\begin{aligned}
W_{j, k}= & \sum_{u=1}^{n-1} \rho_{u}\left\{e^{i \lambda_{j} u} T_{k}(u)+e^{-i \lambda_{j} u} T_{k}(-u)\right\} \\
= & \sum_{u=1}^{n-1}\left(\rho_{u}-\rho_{u+1}\right) \sum_{q=1}^{u}\left\{-e^{i \lambda_{j} q} D_{q}\left(\lambda_{k}\right)-e^{i\left(\lambda_{k}-\lambda_{j} q\right)} \overline{D_{q}\left(\lambda_{k}\right)}\right\} \\
& +\rho_{n} \sum_{q=1}^{n-1}\left\{-e^{i \lambda_{j} q} D_{q}\left(\lambda_{k}\right)-e^{i\left(\lambda_{k}-\lambda_{j} q\right)} \overline{D_{q}\left(\lambda_{k}\right)}\right\}
\end{aligned}
$$

implying

$$
\left|W_{j, k}\right| \leq K \sum_{u=1}^{n-1}\left|\rho_{u}-\rho_{u+1}\right|\left|\sum_{q=1}^{u} e^{i \lambda_{j} q} D_{q}\left(\lambda_{k}\right)\right|+K\left|\rho_{n}\right|\left|\sum_{q=1}^{n-1} e^{i \lambda_{j} q} D_{q}\left(\lambda_{k}\right)\right|
$$


Since

$$
\begin{aligned}
\sum_{q=1}^{u} e^{i \lambda_{j} q} D_{q}\left(\lambda_{k}\right) & =\sum_{q=1}^{u} e^{i \lambda_{j} q} \sum_{t=1}^{q} e^{i t \lambda_{k}}=\sum_{q=1}^{u} e^{i \lambda_{j} q} \frac{1-e^{i q \lambda_{k}}}{e^{-i \lambda_{k}}-1} \\
& =\frac{1}{e^{-i \lambda_{k}}-1} \sum_{q=1}^{u}\left(e^{i \lambda_{j} q}-e^{i \lambda_{j+k} q}\right)=\frac{D_{u}\left(\lambda_{j}\right)-D_{u}\left(\lambda_{j+k}\right)}{e^{-i \lambda_{k}}-1},
\end{aligned}
$$

and $\left|e^{-i \lambda}-1\right| \sim|\lambda|$ as $\lambda \rightarrow 0$, we have

$$
\left|\sum_{q=1}^{u} e^{i \lambda_{j} q} D_{q}\left(\lambda_{k}\right)\right| \leq K \frac{n}{|k|}\left\{\left|D_{u}\left(\lambda_{j}\right)\right|+\left|D_{u}\left(\lambda_{j+k}\right)\right|\right\}
$$

So, using (C.3) and for $a=\min \{|j|,|j+k|\}$,

$$
\begin{aligned}
\left|W_{j, k}\right| & \leq K \frac{n}{|k|} \sum_{u=1}^{[n / a]}\left|\rho_{u+1}\right|+K \frac{n}{|k|} \sum_{u=[n / a]+1}^{n-1} \frac{\left|\rho_{u+1}\right|}{u} \frac{n}{a}+K\left|\rho_{n}\right| \frac{n^{2}}{|k| a} \\
& \leq K \frac{n}{|k|} \sum_{u=1}^{[n / a]} u^{-\frac{1}{2}}+K \frac{n^{2}}{|k| a} \sum_{u=[n / a]+1}^{n-1} u^{-\frac{3}{2}}+K \frac{n^{\frac{3}{2}}}{|k| a} \\
& \leq K \frac{n}{|k|}\left(\frac{n}{a}\right)^{\frac{1}{2}}+K \frac{n^{2}}{|k| a}\left(\frac{n}{a}\right)^{-\frac{1}{2}}+K \frac{n^{\frac{3}{2}}}{|k| a} \leq K \frac{n^{\frac{3}{2}}}{|k| a^{\frac{1}{2}}},
\end{aligned}
$$

yielding

$$
\begin{array}{cl}
\left|W_{j, k-j} W_{k, j-k}\right| \leq K \frac{n^{3}}{(j-k)^{2} \min \{j, k\}}, & 1 \leq j, k \leq m, j \neq k, \\
\left|W_{j,-j-k} W_{-k, j+k}\right| \leq K \frac{n^{3}}{(j+k)^{2} \min \{j, k\}}, & 1 \leq j, k \leq m .
\end{array}
$$

Thus, using (C.52), (C.53), (C.54) in (C.51),

$$
\operatorname{Var}\left\{\hat{F}_{V V}\left(\lambda_{m}\right)\right\} \leq \frac{K}{n^{4}} \sum_{j=1}^{m} \frac{n^{3}}{j}+\frac{K}{n^{4}} \sum_{\substack{j, k=1 \\ j \neq k}}^{m} \frac{n^{3}}{(j-k)^{2} \min \{j, k\}}+\frac{K}{n^{4}} \sum_{j, k=1}^{m} \frac{n^{3}}{(j+k)^{2} \min \{j, k\}}
$$




$$
\begin{aligned}
& \leq K \frac{\log m}{n}+\frac{K}{n^{4}} \sum_{\substack{j, k=1 \\
j<k}}^{m} \frac{n^{3}}{(j-k)^{2} j}+\frac{K}{n^{4}} \sum_{\substack{j, k=1 \\
j \leq k}}^{m} \frac{n^{3}}{(j+k)^{2} j} \\
& \leq K \frac{\log m}{n}+\frac{K}{n} \sum_{j=1}^{m-1} j^{-1} \sum_{a=1}^{m-j} a^{-2}+\frac{K}{n} \sum_{j=1}^{m} j^{-1} \sum_{a=2 j}^{m+j} a^{-2} \leq K \frac{\log m}{n} .
\end{aligned}
$$

